\title{
Weighted piecewise pseudo almost automorphic functions with applications to abstract impulsive $\nabla$-dynamic equations on time scales
}

\author{
Chao Wang $^{1 *}$ and Ravi P Agarwal ${ }^{2,3}$
}

"Correspondence:

chaowang@ynu.edu.cn

${ }^{1}$ Department of Mathematics,

Yunnan University, Kunming,

Yunnan 650091, People's Republic

of China

Full list of author information is

available at the end of the article

\begin{abstract}
In the present paper, by introducing the concept of equipotentially almost automorphic sequence, the concept of weighted piecewise pseudo almost automorphic functions on time scales is proposed. Some first results about their basic properties are obtained and some composition theorems are established. Then we apply these to investigate the existence of weighted piecewise pseudo almost automorphic mild solutions to abstract impulsive $\nabla$-dynamic equations on time scales. In addition, the exponential stability of weighted piecewise pseudo almost automorphic mild solutions is also considered. Finally, the obtained results are applied to the study of a class of $\nabla$-partial differential equations on time scales.
\end{abstract} MSC: 34N05; 35B15; 43A60; 12H20; 35R12

Keywords: time scales; weighted piecewise pseudo almost automorphic functions; abstract impulsive $\nabla$-dynamic equations; weighted piecewise pseudo almost automorphic mild solutions

\section{Introduction}

Almost automorphic functions, which are more general than almost periodic functions, were introduced by Bochner in relation to some aspects of differential geometry (see [1$3]$ ). For more details as regards this topic we refer to the recent books [4-6], where the authors gave important overviews about the theory of almost automorphic functions and their applications to differential equations. Almost automorphic and pseudo almost automorphic solutions in the context of differential equations had been studied by several authors [7-21]. N'Guérékata [13] and Xiao [15, 21] with their collaborators established the existence and uniqueness theorems of pseudo almost automorphic solutions to some semilinear abstract differential equations. Recently, Blot et al. [22] introduced the concept of weighted pseudo almost automorphic functions, which generalizes the concept of weighted pseudo almost periodicity [23-26], and the author proved some interesting properties of the space of weighted pseudo almost automorphic functions like the completeness and the composition theorem, which have many applications in the context of differential equations. For other contributions to the study of weighted pseudo almost automorphy, we refer the reader to [27-30] and references therein.

@2014 Wang and Agarwal; licensee Springer. This is an Open Access article distributed under the terms of the Creative Commons Attribution License (http://creativecommons.org/licenses/by/2.0), which permits unrestricted use, distribution, and reproduction in any medium, provided the original work is properly cited. 
On the other hand, the theory of time scales, which has recently received a lot of attention, was introduced by Hilger in his PhD thesis in 1988 [31] in order to unify continuous and discrete analysis. This theory represents a powerful tool for applications to economics, population models, and quantum physics among others. In fact, the progressive field of dynamic equations on time scales contains links to and extends the classical theory of differential and difference equations. For instance, by choosing the time scale to be the set of real numbers, the general result yields a result for differential equations. In a similar way, by choosing the time scale to be the set of integers, the same general result yields a result for difference equations. However, since there are many other time scales than just the set of real numbers or the set of integers, one has a much more general result. For these reasons, based on the concept of almost periodic time scales proposed in [32,33], the concept of weighted pseudo almost automorphic functions on almost periodic time scales was formally introduced by Wang and Li (2013) in [34]. Moreover, some first results were proven which concern the weighted pseudo almost automorphic mild solution to abstract $\Delta$-dynamic equations on time scales. In addition, by using the results obtained in [32, 33], Lizama and Mesquita [35] presented some new results about basic properties of almost automorphic functions on time scales and proved the existence and uniqueness of an almost automorphic solution to a class of $\Delta$-dynamic equations.

For another thing, many phenomena in nature are characterized by the fact that their states are subject to sudden changes at certain moments and therefore can be described by impulsive system (see [36, 37]). Many evolution processes, optimal control models in economics, stimulated neural networks, population models, artificial intelligence, and robotics are characterized by the fact that at certain moments of time they undergo abrupt changes of state. The existence of almost periodic solutions of abstract impulsive differential equations has been considered by many authors; see [38-41].

However, to the best of our knowledge, the concept of weighted piecewise pseudo almost automorphic functions on time scales has not been introduced in any literature until now, so there was no work on discussing weighted piecewise pseudo almost automorphic problems of impulsive dynamic equations on time scales before. Therefore, in this paper, by introducing the concept of equipotentially almost automorphic sequence, the concept of weighted piecewise pseudo almost automorphic functions on time scales is proposed. The first results about their basic properties are obtained and some composition theorems are established. Then we apply these composition theorems to investigate the existence of weighted piecewise pseudo almost automorphic mild solutions to abstract impulsive $\nabla$-dynamic equations as follows:

$$
\left\{\begin{array}{l}
x^{\nabla}(t)=A(t) x^{\varrho}+f(t, x(t)), \quad t \in \mathbb{T}, t \neq t_{i}, i \in \mathbb{Z}, \\
\Delta x\left(t_{i}\right)=x\left(t_{i}^{+}\right)-x\left(t_{i}^{-}\right)=I_{i}\left(x\left(t_{i}\right)\right), \quad t=t_{i},
\end{array}\right.
$$

where $A \in P C_{\mathrm{ld}}(\mathbb{T}, \mathbb{X})$ is a linear operator in the Banach space $\mathbb{X}$ and $f \in P C_{\mathrm{ld}}(\mathbb{T} \times \mathbb{X}, \mathbb{X})$, $x^{\varrho}=x(\varrho(t)) . f, I_{i}, t_{i}$ satisfy suitable conditions that will be established later and $\mathbb{T}$ is an almost periodic time scale. In addition, the notations $x\left(t_{i}^{+}\right)$and $x\left(t_{i}^{-}\right)$represent the righthand and the left-hand side limits of $x(\cdot)$ at $t_{i}$, respectively. In addition, some useful lemmas are obtained and the exponential stability of weighted piecewise pseudo almost automorphic mild solutions is also considered. Finally, we apply these obtained results to study a class of $\nabla$-partial differential equations on time scales. 


\section{Preliminaries}

In the following, we will introduce some basic knowledge of time scales which is very useful to the proof of our relative results.

A time scale $\mathbb{T}$ is a closed subset of $\mathbb{R}$. It follows that the jump operators $\sigma, \varrho: \mathbb{T} \rightarrow \mathbb{T}$ defined by $\sigma(t)=\inf \{s \in \mathbb{T}: s>t\}$ and $\varrho(t)=\sup \{s \in \mathbb{T}: s<t\}$ (supplemented by inf $\phi:=$ $\sup \mathbb{T}$ and $\sup \phi:=\inf \mathbb{T}$ ) are well defined. The point $t \in \mathbb{T}$ is left-dense, left-scattered, rightdense, right-scattered if $\varrho(t)=t, \varrho(t)<t, \sigma(t)=t, \sigma(t)>t$, respectively. If $\mathbb{T}$ has a rightscattered minimum $m$, define $\mathbb{T}_{k}:=\mathbb{T} \backslash m$; otherwise, set $\mathbb{T}^{k}=\mathbb{T}$. By the notations $[a, b]_{\mathbb{T}}$, $[a, b)_{\mathbb{T}}$ and so on, we will denote time scale intervals

$$
[a, b]_{\mathbb{T}}=\{t \in \mathbb{T}: a \leq t \leq b\}
$$

where $a, b \in \mathbb{T}$ with $a<\varrho(b)$.

The graininess function is defined by $v: \mathbb{T} \rightarrow[0, \infty): v(t):=t-\varrho(t)$, for all $t \in \mathbb{T}$.

Definition 2.1 ([42]) The function $f: \mathbb{T} \rightarrow \mathbb{R}$ is called ld-continuous provided that it is continuous at each left-dense point and has a right-sided limit at each point, write $f \in$ $C_{\text {ld }}(\mathbb{T})=C_{\text {ld }}(\mathbb{T}, \mathbb{R})$. Let $t \in \mathbb{T}_{k}$, the Delta derivative of $f$ at $t$ such that

$$
\left|f(\varrho(t))-f(s)-f^{\nabla}(t)[\varrho(t)-s]\right| \leq \varepsilon|\varrho(t)-s|
$$

for all $s \in U$, at fixed $t$. Let $F$ be a function, it is called the antiderivative of $f: \mathbb{T} \rightarrow \mathbb{R}$ provided $F^{\nabla}(t)=f(t)$ for each $t \in \mathbb{T}_{k}$. If $F^{\nabla}(t)=f(t)$, then we define the delta integral by

$$
\int_{a}^{t} f(s) \nabla s=F(t)-F(a)
$$

Definition $2.2([42])$ A function $p: \mathbb{T} \rightarrow \mathbb{R}$ is called $v$-regressive provided $1-v(t) p(t) \neq 0$ for all $t \in \mathbb{T}_{k}$. The set of all regressive and ld-continuous functions $p: \mathbb{T} \rightarrow \mathbb{R}$ will be denoted by $\mathcal{R}_{v}=\mathcal{R}_{v}(\mathbb{T})=\mathcal{R}_{v}(\mathbb{T}, \mathbb{R})$. We define the set $\mathcal{R}_{v}^{+}=\mathcal{R}_{v}^{+}(\mathbb{T}, \mathbb{R})=\left\{p \in \mathcal{R}_{v}: 1-v(t) p(t)>\right.$ $0, \forall t \in \mathbb{T}\}$.

Definition 2.3 ([42]) If $r$ is a regressive function, then the generalized exponential function $\hat{e}_{r}$ is defined by

$$
\hat{e}_{r}(t, s)=\exp \left\{\int_{s}^{t} \hat{\xi}_{v(\tau)}(r(\tau)) \nabla \tau\right\}
$$

for all $s, t \in \mathbb{T}$, where the $v$-cylinder transformation is as in

$$
\hat{\xi}_{h}(z):=-\frac{1}{h} \log (1-z h) .
$$

Lemma 2.1 ([42]) Assume that $p, q: \mathbb{T} \rightarrow \mathbb{R}$ are two v-regressive functions, then

(i) $\hat{e}_{0}(t, s) \equiv 1$ and $\hat{e}_{p}(t, t) \equiv 1$;

(ii) $\hat{e}_{p}(\varrho(t), s)=(1-v(t) p(t)) \hat{e}_{p}(t, s)$;

(iii) $\hat{e}_{p}(t, s)=\frac{1}{\hat{e}_{p}(s, t)}=e_{\ominus v}(s, t)$;

(iv) $\hat{e}_{p}(t, s) \hat{e}_{p}(s, r)=\hat{e}_{p}(t, r)$;

(v) $\left(\hat{e}_{\ominus_{\nu} p}(t, s)\right)^{\nabla}=\left(\ominus_{\nu} p\right)(t) \hat{e}_{\ominus_{\nu} p}(t, s)$. 
Lemma 2.2 ([43]) For each $t_{0} \in \mathbb{T}$ in $\mathbb{T} \backslash \mathbb{T}_{k}$ the single-point set $\left\{t_{0}\right\}$ is $\nabla$-measurable and its $\nabla$-measure is given by $\mu_{\nabla}\left(\left\{t_{0}\right\}\right)=t_{0}-\varrho\left(t_{0}\right)$.

Lemma 2.3 ([43]) If $a, b \in \mathbb{T}$ and $a \leq b$, then

$$
\begin{aligned}
& \mu_{\nabla}\left((a, b]_{\mathbb{T}}\right)=b-a, \quad \mu_{\nabla}\left((a, b)_{\mathbb{T}}\right)=\varrho(b)-a . \\
& \text { If } a, b \in \mathbb{T} \backslash \mathbb{T}^{k} \text { and } a \leq b, \text { then } \\
& \mu_{\nabla}\left([a, b)_{\mathbb{T}}\right)=\varrho(b)-\varrho(a), \quad \mu_{\nabla}\left([a, b]_{\mathbb{T}}\right)=b-\varrho(a) .
\end{aligned}
$$

For more details of time scales and $\nabla$-measurability, one is referred to [42, 43]. For more on time scales, see [44-49].

Definition 2.4 ([32-34]) A time scale $\mathbb{T}$ is called an almost periodic time scale if

$$
\Pi:=\{\tau \in \mathbb{R}: t \pm \tau \in \mathbb{T}, \forall t \in \mathbb{T}\} \neq\{0\}
$$

Remark 2.4 Definition 3.1 introduced in [35] is the same as the concept of almost periodic time scales proposed in $[32,34]$, and $\mathbb{T}$ is also called an invariant time scale under translations in [35].

After these preparations, in the next section, we will introduce the concept of weighted piecewise pseudo almost automorphic functions on time scales in a Banach space and some of their basic properties are investigated.

\section{Weighted piecewise pseudo almost automorphic functions on time scales}

In the following, we will give the definition of ld-piecewise continuous functions on time scales.

Definition 3.1 We say $\varphi: \mathbb{T} \rightarrow \mathbb{X}$ is ld-piecewise continuous with respect to a sequence $\left\{\tau_{i}\right\} \subset \mathbb{T}$ which satisfy $\tau_{i}<\tau_{i+1}, i \in \mathbb{Z}$, if $\varphi(t)$ is continuous on $\left(\tau_{i}, \tau_{i+1}\right]_{\mathbb{T}}$ and ld-continuous on $\mathbb{T} \backslash\left\{\tau_{i}\right\}$. Furthermore, $\left(\tau_{i}, \tau_{i+1}\right]_{\mathbb{T}}$ are called intervals of continuity of the function $\varphi(t)$.

For convenience, $P C_{\mathrm{ld}}(\mathbb{T}, \mathbb{X})$ denotes the set of all ld-piecewise continuous functions with respect to a sequence $\left\{\tau_{i}\right\}, i \in \mathbb{Z}$. Similar to Definition 3.1, we can also introduce the concept of functions which belong to $P C_{\mathrm{rd}}(\mathbb{T}, \mathbb{X})$.

Throughout the paper, we denote by $\mathbb{X}$ a Banach space; let $\mathfrak{B}$ be the set consisting of all sequences $\left\{t_{i}\right\}_{i \in \mathbb{Z}}$ such that $\theta=\inf _{i \in \mathbb{Z}}\left(t_{i+1}-t_{i}\right)>0$. For $\left\{t_{i}\right\}_{i \in \mathbb{Z}} \in \mathfrak{B}$, let $B P C_{\mathrm{ld}}(\mathbb{T}, \mathbb{X})$ be the space formed by all bounded ld-piecewise continuous functions $\phi: \mathbb{T} \rightarrow \mathbb{X}$ such that $\phi(\cdot)$ is continuous at $t$ for any $t \notin\left\{t_{i}\right\}_{i \in \mathbb{Z}}$ and $\phi\left(t_{i}\right)=\phi\left(t_{i}^{-}\right)$for all $i \in \mathbb{Z}$; let $\Omega$ be a subset of $\mathbb{X}$ and let $B P C_{\mathrm{ld}}(\mathbb{T} \times \Omega, \mathbb{X})$ be the space formed by all bounded piecewise continuous functions $\phi: \mathbb{T} \times \Omega \rightarrow \mathbb{X}$ such that, for any $x \in \Omega, \phi(\cdot, x) \in B P C_{\mathrm{ld}}(\mathbb{T} \times \mathbb{X}, \mathbb{X})$. For any $t \in \mathbb{T}, \phi(t, \cdot)$ is continuous at $x \in \Omega$.

Let $U P C(\mathbb{T}, \mathbb{X})$ be the space of all functions $\varphi \in P C_{\mathrm{ld}}(\mathbb{T}, \mathbb{X})$ such that $\phi$ satisfies the condition: for any $\varepsilon>0$, there exists a positive number $\delta=\delta(\varepsilon)$ such that if the points $t^{\prime}, t^{\prime \prime}$ belong to the same interval of continuity of $\varphi$ and $\left|t^{\prime}-t^{\prime \prime}\right|<\delta$ implies $\left\|\varphi\left(t^{\prime}\right)-\varphi\left(t^{\prime \prime}\right)\right\|<\varepsilon$. 
Now, we introduce the set

$$
\mathfrak{B}=\left\{\left\{t_{k}\right\}: t_{k} \in \mathbb{T}, t_{k}<t_{k+1}, k \in \mathbb{Z}, \lim _{t \rightarrow \pm \infty}= \pm \infty\right\},
$$

which denotes all unbounded increasing sequences of real numbers. Let $T, P \in \mathfrak{B}$ and let $s(T \cup P): \mathfrak{B} \rightarrow \mathfrak{B}$ be a map such that the set $s(T \cup P)$ forms a strictly increasing sequence. For $D \subset \mathbb{R}$ and $\varepsilon>0$, we introduce the notations $\theta_{\varepsilon}(D)=\{t+\varepsilon: t \in D\}, F_{\varepsilon}(D)=\bigcap_{\varepsilon}\left\{\theta_{\varepsilon}(D)\right\}$. Denote by $\tilde{\phi}=(\varphi(t), T)$ the element from the space $P C_{\mathrm{ld}}(\mathbb{T}, \mathbb{X}) \times \mathfrak{B}$. For every sequence of real numbers $\left\{s_{n}\right\}, n=1,2, \ldots$ with $\theta_{s_{n}} \tilde{\phi}:=\left(\varphi\left(t+s_{n}\right), T-s_{n}\right)$, we shall consider the sets $\left\{\varphi\left(t+s_{n}\right), T-s_{n}\right\} \subset P C_{\text {ld }} \times \mathfrak{B}$, where

$$
T-s_{n}=\left\{t_{k}-s_{n}: k \in \mathbb{Z}, n=1,2, \ldots\right\} .
$$

Definition 3.2 Let $\left\{t_{i}\right\} \in \mathfrak{B}, i \in \mathbb{Z}$. We say $\left\{t_{i}^{j}\right\}$ is a derivative sequence of $\left\{t_{i}\right\}$ and

$$
t_{i}^{j}=t_{i+j}-t_{i}, \quad i, j \in \mathbb{Z}
$$

Definition 3.3 Let $t_{i}^{j}=t_{i+j}-t_{i}, i, j \in \mathbb{Z}$. We say $\left\{t_{i}^{j}\right\}, i, j \in \mathbb{Z}$, is equipotentially almost automorphic on an almost periodic time scale $\mathbb{T}$ if, for any sequence $\left\{s_{n}\right\} \subset \mathbb{Z}$, there exists a subsequence $\left\{s_{n}^{\prime}\right\}$ such that

$$
\lim _{n \rightarrow \infty} t_{k}^{s_{n}^{\prime}}=\gamma_{k}
$$

is well defined for each $k \in \mathbb{Z}$ and

$$
\lim _{n \rightarrow \infty} \gamma_{k}^{-s_{n}^{\prime}}=t_{k}
$$

for each $k \in \mathbb{Z}$.

Definition 3.4 A function $\phi \in P C_{\mathrm{ld}}(\mathbb{T}, \mathbb{X})$ is said to be ld-piecewise almost automorphic if the following conditions are fulfilled:

(i) $T=\left\{t_{k}\right\}$ is an equipotentially almost automorphic sequence.

(ii) Let $\varphi \in P C_{\mathrm{ld}}(\mathbb{T}, \mathbb{X})$ be a bounded function with respect to a sequence $T=\left\{t_{k}\right\}$. We say that $\varphi$ is piecewise almost automorphic if from every sequence $\left\{s_{n}\right\}_{n=1}^{\infty} \subset \Pi$, we can extract a subsequence $\left\{\tau_{n}\right\}_{n=1}^{\infty}$ such that

$$
\tilde{\phi}^{*}=\left(\varphi^{*}(t), T^{*}\right)=\lim _{n \rightarrow \infty}\left(\varphi\left(t+\tau_{n}\right), T-\tau_{n}\right)=\lim _{n \rightarrow \infty} \theta_{\tau_{n}} \tilde{\phi}
$$

is well defined for each $t \in \mathbb{T}$ and

$$
\tilde{\phi}=(\varphi(t), T)=\lim _{n \rightarrow \infty}\left(\varphi^{*}\left(t-\tau_{n}\right), T^{*}+\tau_{n}\right)=\lim _{n \rightarrow \infty} \theta_{-\tau_{n}} \tilde{\phi}^{*}
$$

for each $t \in \mathbb{T}$. Denote by $A A(\mathbb{T}, \mathbb{X})$ the set of all such functions.

(iii) A bounded function $f \in P C_{\mathrm{ld}}(\mathbb{T} \times \mathbb{X}, \mathbb{X})$ with respect to a sequence $T=\left\{t_{k}\right\}$ is said to be piecewise almost automorphic if $f(t, x)$ is piecewise automorphic in $t \in \mathbb{T}$ uniformly in $x \in B$, where $B$ is any bounded subset of $\mathbb{X}$. Denote by $A A(\mathbb{T} \times \mathbb{X}, \mathbb{X})$ the set of all such functions. 
Similarly, we can also introduce the concept of piecewise almost automorphic functions which belong to $P C_{\mathrm{rd}}(\mathbb{T}, \mathbb{X})$.

Let $U$ be the set of all functions $\rho: \mathbb{T} \rightarrow(0, \infty)$ which are positive and locally $\nabla$ integrable over $\mathbb{T}$. For a given $r \in[0, \infty) \cap \Pi$ and $\forall t_{0} \in \mathbb{T}$, set

$$
m\left(r, \rho, t_{0}\right):=\int_{t_{0}-r}^{t_{0}+r} \rho(s) \nabla s
$$

for each $\rho \in U$.

Remark 3.1 In (2), if $\mathbb{T}=\mathbb{R}, t_{0}=0$, one can easily get

$$
m\left(r, \rho, t_{0}\right):=\int_{-r}^{r} \rho(s) \mathrm{d} s
$$

if $\mathbb{T}=\mathbb{Z}, t_{0}=0$, one has the following:

$$
m(r, \rho)=\sum_{k=-r+1}^{r} \rho(k) .
$$

Define

$$
\begin{aligned}
& U_{\infty}:=\left\{\rho \in U: \lim _{r \rightarrow \infty} m\left(r, \rho, t_{0}\right)=\infty\right\}, \\
& U_{B}:=\left\{\rho \in U_{\infty}: \rho \text { is bounded and } \inf _{s \in \mathbb{T}} \rho(s)>0\right\} .
\end{aligned}
$$

It is clear that $U_{B} \subset U_{\infty} \subset U$. Now for $\rho \in U_{\infty}$ define

$$
\begin{aligned}
P A A_{0}(\mathbb{T}, \rho):= & \left\{\phi \in B P C_{\mathrm{ld}}(\mathbb{T}, \mathbb{X}): \lim _{r \rightarrow \infty} \frac{1}{m\left(r, \rho, t_{0}\right)} \int_{t_{0}-r}^{t_{0}+r}\|\phi(s)\| \rho(s) \nabla s=0,\right. \\
& \left.\forall t_{0} \in \mathbb{T}, r \in \Pi\right\} .
\end{aligned}
$$

Similarly, we define

$$
\begin{aligned}
P A A_{0}(\mathbb{T} \times \mathbb{X}, \rho):= & \left\{\Phi \in B P C_{\mathrm{ld}}(\mathbb{T} \times \Omega, \mathbb{X}):\right. \\
& \lim _{r \rightarrow \infty} \frac{1}{m\left(r, \rho, t_{0}\right)} \int_{t_{0}-r}^{t_{0}+r}\|\Phi(s, x)\| \rho(s) \nabla s=0 \\
& \text { uniformly with respect to } \left.x \in K, \forall t_{0} \in \mathbb{T}, r \in \Pi\right\} .
\end{aligned}
$$

We are now ready to introduce the sets $W P A A(\mathbb{T}, \rho)$ and $W P A A(\mathbb{T} \times \mathbb{X}, \rho)$ of weighted pseudo almost periodic functions:

$$
\begin{aligned}
W P A A(\mathbb{T}, \rho)=\{f= & \left.g+\phi \in P C_{\mathrm{ld}}(\mathbb{T}, \mathbb{X}): g \in A A(\mathbb{T}, \mathbb{X}) \text { and } \phi \in P A A_{0}(\mathbb{T}, \rho)\right\}, \\
W P A A(\mathbb{T} \times \mathbb{X}, \rho)= & \left\{f=g+\phi \in P C_{\mathrm{ld}}(\mathbb{T} \times \mathbb{X}, \mathbb{X}): g \in A A(\mathbb{T} \times \mathbb{X}, \mathbb{X})\right. \\
& \text { and } \left.\phi \in P A A_{0}(\mathbb{T} \times \mathbb{X}, \rho)\right\} .
\end{aligned}
$$


Lemma 3.2 Let $\phi \in B P C_{\mathrm{ld}}(\mathbb{T}, \mathbb{X})$. Then $\phi \in P A A_{0}(\mathbb{T}, \rho)$ where $\rho \in U_{B}$ if and only if, for every $\varepsilon>0$,

$$
\lim _{r \rightarrow \infty} \frac{1}{m\left(r, \rho, t_{0}\right)} \mu_{\nabla}\left(M_{r, \varepsilon, t_{0}}(\phi)\right)=0,
$$

where $r \in \Pi$ and $M_{r, \varepsilon, t_{0}}(\phi):=\left\{t \in\left[t_{0}-r, t_{0}+r\right]_{\mathbb{T}}:\|\phi(t)\| \geq \varepsilon\right\}$.

Proof (a) Necessity. For contradiction, suppose that there exists $\varepsilon_{0}>0$ such that

$$
\lim _{r \rightarrow \infty} \frac{1}{m\left(r, \rho, t_{0}\right)} \mu_{\nabla}\left(M_{r, \varepsilon_{0}, t_{0}}(\phi)\right) \neq 0 .
$$

Then there exists $\delta>0$ such that, for every $n \in \mathbb{N}$,

$$
\frac{1}{m\left(r_{n}, \rho, t_{0}\right)} \mu_{\nabla}\left(M_{r_{n}, \varepsilon_{0}, t_{0}}(\phi)\right) \geq \delta \quad \text { for some } r_{n}>n \text {, where } r_{n} \in \Pi \text {. }
$$

So we get

$$
\begin{aligned}
\frac{1}{m\left(r_{n}, \rho, t_{0}\right)} \int_{t_{0}-r}^{t_{0}+r}\|\phi(s)\| \rho(s) \nabla s= & \frac{1}{m\left(r_{n}, \rho, t_{0}\right)} \int_{M_{r_{n}, \varepsilon_{0}, t_{0}}(\phi)}\|\phi(s)\| \rho(s) \nabla s \\
& +\frac{1}{m\left(r_{n}, \rho, t_{0}\right)} \\
& \times \int_{\left[t_{0}-r_{0}, t_{0}+r\right]_{\mathbb{T}} \backslash M_{r_{n}, \varepsilon_{0}, t_{0}}(\phi)}\|\phi(s)\| \rho(s) \nabla s \\
\geq & \frac{1}{m\left(r_{n}, \rho, t_{0}\right)} \int_{M_{r_{n}, \varepsilon_{0}, t_{0}}(\phi)}\|\phi(s)\| \rho(s) \nabla s \\
\geq & \frac{\varepsilon_{0}}{m\left(r_{n}, \rho, t_{0}\right)} \int_{M_{r_{n}, \varepsilon_{0}, t_{0}}(\phi)}\|\phi(s)\| \rho(s) \nabla s \\
\geq & \varepsilon_{0} \delta \gamma,
\end{aligned}
$$

where $\gamma=\inf _{s \in \mathbb{T}} \rho(s)$. This contradicts the assumption.

(b) Sufficiency. Assume that $\lim _{r \rightarrow \infty} \frac{1}{m\left(r, \rho, t_{0}\right)} \mu_{\nabla}\left(M_{r, \varepsilon, t_{0}}(\phi)\right)=0$. Then for every $\varepsilon>0$, there exists $r_{0}>0$ such that, for every $r>r_{0}$,

$$
\frac{1}{m\left(r, \rho, t_{0}\right)} \mu_{\nabla}\left(M_{r, \varepsilon, t_{0}}(\phi)\right)<\frac{\varepsilon}{K M}
$$

where $M:=\sup _{t \in \mathbb{T}}\|\phi(t)\|<\infty$ and $K:=\sup _{t \in \mathbb{T}} \rho(t)<\infty$.

Now, we have

$$
\begin{aligned}
\frac{1}{m\left(r, \rho, t_{0}\right)} \int_{t_{0}-r}^{t_{0}+r}\|\phi(s)\| \rho(s) \nabla s= & \frac{1}{m\left(r, \rho, t_{0}\right)}\left(\int_{M_{r, s, t_{0}}(\phi)}\|\phi(s)\| \rho(s) \nabla s\right. \\
& \left.+\int_{\left[t_{0}-r, t_{0}+r\right]_{\mathbb{T}} \backslash M_{r, \varepsilon, t_{0}}(\phi)}\|\phi(s)\| \rho(s) \nabla s\right) \\
\leq & \frac{M K}{m\left(r, \rho, t_{0}\right)} \mu_{\nabla}\left(M_{r, \varepsilon, t_{0}}(\phi)\right)
\end{aligned}
$$




$$
\begin{aligned}
& \quad+\frac{\varepsilon}{m\left(r, \rho, t_{0}\right)} \int_{\left[t_{0}-r, t_{0}+r\right]_{\mathbb{T}} \backslash M_{r, \varepsilon, t_{0}}(\phi)} \rho(s) \nabla s \\
& \leq 2 \varepsilon .
\end{aligned}
$$

Therefore, $\lim _{r \rightarrow \infty} \frac{1}{m\left(r, \rho, t_{0}\right)} \int_{t_{0}-r}^{t_{0}+r}\|\phi(s)\| \rho(s) \nabla s=0$, that is, $\phi \in P A A_{0}(\mathbb{T}, \rho)$. This completes the proof.

Lemma 3.3 $P A A_{0}(\mathbb{T}, \rho)$ is a translation invariant set of $B P C_{\mathrm{ld}}(\mathbb{T}, \mathbb{X})$ with respect to $\Pi$ if $\rho \in U_{B}$, i.e., for any $s \in \Pi$, one has $\phi(t+s):=\theta_{s} \phi \in P A A_{0}(\mathbb{T}, \rho)$ if $\rho \in U_{B}$.

Proof For any $s \in \Pi, \phi \in P A A_{0}(\mathbb{T}, \rho), \varepsilon>0, r>0$, we have

$$
\begin{aligned}
M_{r, \varepsilon, t_{0}}\left(T_{s} \phi\right) & =\left\{t \in\left[t_{0}-r, t_{0}+r\right]_{\mathbb{T}}:\left\|T_{s}(t)\right\| \geq \varepsilon\right\} \\
& =\left\{t \in\left[t_{0}-r, t_{0}+r\right]_{\mathbb{T}}:\|\phi(t+s)\| \geq \varepsilon\right\} \\
& =\left\{t \in\left[t_{0}-r+s, t_{0}+r+s\right]_{\mathbb{T}}:\|\phi(t)\| \geq \varepsilon\right\} \\
& \subseteq\left\{t \in\left[t_{0}-r-|s|, t_{0}+r+|s|\right]_{\mathbb{T}}:\|\phi(t)\| \geq \varepsilon\right\} .
\end{aligned}
$$

Hence

$$
\begin{aligned}
\frac{1}{m\left(r, \rho, t_{0}\right)} \mu_{\nabla}\left(M_{r, \varepsilon, t_{0}}\left(T_{s} \phi\right)\right) & \leq \frac{1}{m\left(r, \rho, t_{0}\right)} \mu_{\nabla}\left(M_{r+|s|, \varepsilon, t_{0}}\left(T_{s} \phi\right)\right) \\
& =\frac{m\left(r+|s|, \rho, t_{0}\right)}{m\left(r, \rho, t_{0}\right)} \frac{1}{m\left(r+|s|, \rho, t_{0}\right)} \mu_{\nabla}\left(M_{r+|s|, \varepsilon, t_{0}}(\phi)\right) .
\end{aligned}
$$

Since $\phi \in P A A_{0}(\mathbb{T}, \rho)$, by Lemma 3.2, we have

$$
\frac{1}{m\left(r+|s|, \rho, t_{0}\right)}\left(M_{r+|s|, \varepsilon, t_{0}}(\phi)\right) \rightarrow 0, \quad r \rightarrow \infty
$$

Furthermore, $\lim _{r \rightarrow \infty} \frac{m\left(r+|s|, \rho, t_{0}\right)}{m\left(r, \rho, t_{0}\right)}=1$, thus

$$
\frac{1}{m\left(r, \rho, t_{0}\right)} \mu_{\nabla}\left(M_{r, \varepsilon, t_{0}}\left(T_{s}(\phi)\right)\right) \rightarrow 0, \quad r \rightarrow \infty
$$

Again, using Lemma 3.2, one can get $\theta_{s} \phi \in P A A_{0}(\mathbb{T}, \rho)$ for any $s \in \Pi$. This completes the proof.

By Definition 3.4, one can easily get the following lemma.

Lemma 3.4 Let $\phi \in A A(\mathbb{T}, \mathbb{X})$, then the range of $\phi, \phi(\mathbb{T})$, is a relatively compact subset of $\mathbb{X}$.

Lemma 3.5 Iff $=g+\phi$ with $g \in A A(\mathbb{T}, \mathbb{X})$, and $\phi \in P A A_{0}(\mathbb{T}, \rho)$, where $\rho \in U_{B}$, then $g(\mathbb{T}) \subset$ $\overline{f(\mathbb{T})}$.

Proof (1) For any $t \in \mathbb{T} \backslash\left\{t_{i}\right\}, g(t) \in g(\mathbb{T})$, one has $g(t)=f(t)-\phi(t)$. Since $g \in A A(\mathbb{T}, \mathbb{X})$, there exists a sequence $\left\{\alpha_{n}\right\} \subset \Pi$ such that $g\left(t+\alpha_{n}\right) \rightarrow g(t), n \rightarrow \infty$. 
Furthermore, by Lemma $3.3, \phi\left(t+\alpha_{n}\right) \in P A A_{0}(\mathbb{T}, \mathbb{X})$, so there exists $\beta \in \Pi$ such that $\phi\left(t+\alpha_{n}+\beta\right) \rightarrow 0, n \rightarrow \infty$. Hence, let $s=t+\beta$, and one has

$$
f\left(s+\alpha_{n}-\beta\right)-\phi\left(t+\alpha_{n}+\beta\right) \rightarrow g(t) \quad \text { for each } t \in \mathbb{T} \text { as } n \rightarrow \infty,
$$

i.e. $f\left(s+\alpha_{n}-\beta\right) \rightarrow g(t)$ for each $t \in \mathbb{T}$ as $n \rightarrow \infty$.

(2) If $\left\{t_{i}\right\} \in \mathfrak{B}$, noting that Definition 3.4, the above sequence $\left\{\alpha_{n}\right\} \subset \Pi$ and the number $\beta \in \Pi$ is suitable for the increasing sequence $\left\{t_{i}\right\}$, so the proof process is the same as (1). This completes the proof.

Lemma 3.6 The decomposition of a weighted piecewise pseudo almost automorphic function according to $A A \oplus P A A_{0}$ is unique for any $\rho \in U_{B}$.

Proof Assume that $f=g_{1}+\phi_{1}$ and $f=g_{2}+\phi_{2}$. Then $\left(g_{1}-g_{2}\right)+\left(\phi_{1}-\phi_{2}\right)=0$. Since $g_{1}-g_{2} \in$ $A A(\mathbb{T}, \mathbb{X})$, and $\phi_{1}-\phi_{2} \in P A A_{0}(\mathbb{T}, \rho)$, in view of Lemma 3.5, we deduce that $g_{1}-g_{2}=0$. Consequently, $\phi_{1}-\phi_{2}=0$, i.e. $\phi_{1}=\phi_{2}$. This completes the proof.

Theorem 3.7 For $\rho \in U_{B},\left(\operatorname{WPAA}(\mathbb{T}, \rho),\|\cdot\|_{\infty}\right)$ is a Banach space.

Proof Assume that $\left\{f_{n}\right\}_{n \in \mathbb{N}}$ is a Cauchy sequence in $\operatorname{WPAA}(\mathbb{T}, \rho)$. We can write uniquely $f_{n}=g_{n}+\phi_{n}$. Using Lemma 3.5, we see that $\left\|g_{p}-g_{q}\right\|_{\infty} \leq\left\|f_{p}-f_{q}\right\|_{\infty}$, from which we deduce that $\left\{g_{n}\right\}_{n \in \mathbb{N}}$ is a Cauchy sequence in $A A(\mathbb{T}, \mathbb{X})$. Hence, $\phi_{n}=f_{n}-g_{n}$ is a Cauchy sequence in $P A A_{0}(\mathbb{T}, \rho)$. We deduce that $g_{n} \rightarrow g \in A A(\mathbb{T}, \mathbb{X}), \phi_{n} \rightarrow \phi \in P A A_{0}(\mathbb{T}, \rho)$, and finally $f_{n} \rightarrow$ $g+\phi \in \operatorname{WPAA}(\mathbb{T}, \rho)$. This completes the proof.

Definition 3.5 Let $\rho_{1}, \rho_{2} \in U_{\infty}$. One says that $\rho_{1}$ is equivalent to $\rho_{2}$, written $\rho_{1} \sim \rho_{2}$ if $\rho_{1} / \rho_{2} \in U_{B}$.

Theorem 3.8 Let $\rho_{1}, \rho_{2} \in U_{\infty}$. If $\rho_{1} \sim \rho_{2}$, then $\operatorname{WPAA}\left(\mathbb{T}, \rho_{1}\right)=\operatorname{WPAA}\left(\mathbb{T}, \rho_{2}\right)$.

Proof Assume that $\rho_{1} \sim \rho_{2}$. There exist $a, b>0$ such that $a \rho_{1} \leq \rho_{2} \leq b \rho_{1}$. So

$$
a m\left(r, \rho_{1}, t_{0}\right) \leq m\left(r, \rho_{2}, t_{0}\right) \leq b m\left(r, \rho_{1}, t_{0}\right),
$$

where $r \in \Pi$ and

$$
\begin{aligned}
\frac{a}{b} \frac{1}{m\left(r, \rho_{1}, t_{0}\right)} \int_{t_{0}-r}^{t_{0}+r}\|\phi(s)\| \rho_{1}(s) \nabla s & \leq \frac{1}{m\left(r, \rho_{2}, t_{0}\right)} \int_{t_{0}-r}^{t_{0}+r}\|\phi(s)\| \rho_{2}(s) \nabla s \\
& \leq \frac{b}{a} \frac{1}{m\left(r, \rho_{1}, t_{0}\right)} \int_{t_{0}-r}^{t_{0}+r}\|\phi(s)\| \rho_{1}(s) \nabla s .
\end{aligned}
$$

This completes the proof.

Lemma 3.9 If $g \in A A(\mathbb{T} \times \mathbb{X}, \mathbb{X})$ and $\alpha \in A A(\mathbb{T}, \mathbb{X})$, then $G(t):=g(\cdot, \alpha(\cdot)) \in A A(\mathbb{T}, \mathbb{X})$.

Proof Let $T=\left\{t_{i}\right\}, \tilde{\phi}=(g(t, x), T) \in A A(\mathbb{T} \times \mathbb{X}, \mathbb{X}) \times \mathfrak{B}$, from every sequence $\left\{s_{n}\right\}_{n=1}^{\infty} \subset \Pi$, we can extract a subsequence $\left\{\tau_{n}\right\}_{n=1}^{\infty}$ such that

$$
\tilde{\phi}^{*}:=\left(g^{*}(t, x), T^{*}\right)=\lim _{n \rightarrow \infty} \theta_{\tau_{n}} \tilde{\phi}=\lim _{n \rightarrow \infty}\left(g\left(t+\tau_{n}, x\right), T-\tau_{n}\right)
$$


uniformly exists on $P C_{\text {ld }}(\mathbb{T} \times \mathbb{X}, \mathbb{X}) \times \mathfrak{B}$. Since $\alpha \in A A(\mathbb{T}, \mathbb{X})$, one can extract $\left\{\tau_{n}^{\prime}\right\} \subset\left\{\tau_{n}\right\}$ such that

$$
\begin{aligned}
\lim _{n \rightarrow \infty} \theta_{\tau_{n}^{\prime}} \tilde{\phi} & =\lim _{n \rightarrow \infty}\left(g\left(t+\tau_{n}^{\prime}, \alpha\left(t+\tau_{n}^{\prime}\right)\right), T-\tau_{n}^{\prime}\right) \\
& =\lim _{n \rightarrow \infty}\left(g\left(t+\tau_{n}^{\prime}, \alpha^{*}(t)\right), T-\tau_{n}^{\prime}\right)=\left(g^{*}\left(t, \alpha^{*}(t)\right), T^{*}\right) .
\end{aligned}
$$

Hence, $G \in A A(\mathbb{T}, \mathbb{X})$. This completes the proof.

Theorem 3.10 Let $f=g+\phi \in W P A A(\mathbb{T} \times \mathbb{X}, \rho)$, where $g \in A A(\mathbb{T} \times \mathbb{X}, \mathbb{X}), \phi \in P A A_{0}(\mathbb{T} \times$ $\mathbb{X}, \rho), \rho \in U_{B}$, and the following conditions hold:

(i) $\{f(t, x): t \in \mathbb{T}, x \in K\}$ is bounded for every bounded subset $K \subseteq \Omega$.

(ii) $f(t, \cdot), g(t, \cdot)$ are uniformly continuous in each bounded subset of $\Omega$ uniformly in $t \in \mathbb{T}$. Then $f(\cdot, h(\cdot)) \in W P A A(\mathbb{T}, \rho)$ if $h \in W P A A(\mathbb{T}, \rho)$ and $h(\mathbb{T}) \subset \Omega$.

Proof We have $f=g+\phi$, where $g \in A A(\mathbb{T} \times \mathbb{X}, \mathbb{X})$ and $\phi \in P A A_{0}(\mathbb{T} \times \mathbb{X}, \rho)$ and $h=\phi_{1}+\phi_{2}$, where $\phi_{1} \in A A(\mathbb{T}, \mathbb{X})$ and $\phi_{2} \in P A A_{0}(\mathbb{T}, \rho)$. Hence, the function $f(\cdot, h(\cdot))$ can be decomposed as

$$
\begin{aligned}
f(\cdot, h(\cdot)) & =g\left(\cdot, \phi_{1}(\cdot)\right)+f(\cdot, h(\cdot))-g\left(\cdot, \phi_{1}(\cdot)\right) \\
& =g\left(\cdot, \phi_{1}(\cdot)\right)+f(\cdot, h(\cdot))-f\left(\cdot, \phi_{1}(\cdot)\right)+\phi\left(\cdot, \phi_{1}(\cdot)\right) .
\end{aligned}
$$

By Lemma 3.9, $g\left(\cdot, \phi_{1}(\cdot)\right) \in A A(\mathbb{T}, \mathbb{X})$. Now, consider the function

$$
\Psi(\cdot):=f(\cdot, h(\cdot))-f\left(\cdot, \phi_{1}(\cdot)\right)
$$

Clearly, $\Psi \in B P C_{\mathrm{ld}}(\mathbb{T}, \mathbb{X})$. For $\Psi$ to be in $P A A_{0}(\mathbb{T}, \rho)$, it is sufficient to show that

$$
\lim _{r \rightarrow \infty} \frac{1}{m\left(r, \rho, t_{0}\right)} \mu_{\nabla}\left(M_{r, \varepsilon, t_{0}}(\Psi)\right)=0 .
$$

Let $K$ be a bounded subset of $\Omega$ such that $\phi(\mathbb{T}) \subseteq K, \phi_{1}(\mathbb{T}) \subseteq K$. By (ii), $f(t, \cdot)$ is uniformly continuous in $\phi(\mathbb{T})$ uniformly in $t \in \mathbb{T}$, and we see that, for given $\varepsilon>0$, there exists $\delta>0$ such that $y_{1}, y_{2} \in K$ and $\left\|y_{1}-y_{2}\right\|<\delta$ implies that

$$
\left\|f\left(t, y_{1}\right)-f\left(t, y_{2}\right)\right\|<\varepsilon, \quad t \in \mathbb{T} .
$$

Thus, for each $t \in \mathbb{T},\left\|\phi_{2}(t)\right\|<\delta$ implies for all $t \in \mathbb{T}$,

$$
\left\|f(t, h(t))-f\left(t, \phi_{1}(t)\right)\right\|<\varepsilon
$$

where $\phi_{2}(t)=h(t)-\phi_{1}(t)$. For $r>0$ and any fixed $t_{0} \in \mathbb{T}$, let $M_{r, \delta, t_{0}}\left(\phi_{2}\right)=\left\{t \in\left[t_{0}-r, t_{0}+r\right]_{\mathbb{T}}\right.$ : $\left.\left\|\phi_{2}\right\| \geq \delta\right\}$, we can obtain

$$
\begin{aligned}
& \frac{1}{m\left(r, \rho, t_{0}\right)} \mu_{\nabla}\left(M_{r, \varepsilon, t_{0}}(\Psi(t))\right) \\
& =\frac{1}{m\left(r, \rho, t_{0}\right)} \mu_{\nabla}\left(M_{r, \varepsilon, t_{0}}\left(f(t, h(t))-f\left(t, \phi_{1}(t)\right)\right)\right)
\end{aligned}
$$




$$
\begin{aligned}
& \leq \frac{1}{m\left(r, \rho, t_{0}\right)} \mu_{\nabla}\left(M_{r, \delta, t_{0}}\left(h(t)-\phi_{1}(t)\right)\right) \\
& =\frac{1}{m\left(r, \rho, t_{0}\right)} \mu_{\nabla}\left(M_{r, \delta, t_{0}}\left(\phi_{2}(t)\right)\right) .
\end{aligned}
$$

Now since $\phi_{2} \in P A A_{0}(\mathbb{T}, \rho)$, Lemma 3.2 yields

$$
\lim _{r \rightarrow \infty} \frac{1}{m\left(r, \rho, t_{0}\right)} \mu_{\nabla}\left(M_{r, \varepsilon, t_{0}}\left(\phi_{2}(t)\right)\right)=0
$$

and this implies that $\Psi \in P A A_{0}(\mathbb{T}, \rho)$.

Finally, we need to show $\phi\left(\cdot, \phi_{1}(\cdot)\right) \in P A A_{0}(\mathbb{T}, \rho)$. Note that $f=g+\phi$ and $g(t, \cdot)$ is uniformly continuous in $\phi_{1}(\mathbb{T})$ uniformly in $t \in \mathbb{T}$. By the assumption (ii), $f(t, \cdot)$ is uniformly continuous in $\phi_{1}(\mathbb{T})$ uniformly in $t \in \mathbb{T}$, so is $\phi$. Since $\phi_{1}(\mathbb{T})$ is relatively compact in $\mathbb{X}$, for $\varepsilon>0$, there exists $\delta>0$ such that $\phi_{1}(\mathbb{T}) \subset \bigcup_{k=1}^{m} B_{k}$, where $B_{k}=\left\{x \in \mathbb{X}:\left\|x-x_{k}\right\|<\delta\right\}$ for some $x_{k} \in \phi_{1}(\mathbb{T})$ and

$$
\left\|\phi\left(t, \phi_{1}(t)\right)-\phi\left(t, x_{k}\right)\right\|<\frac{\varepsilon}{2}, \quad \phi_{1}(t) \in B_{k}, t \in \mathbb{T} .
$$

It is easy to see that the set $U_{k}:=\left\{t \in \mathbb{T}: \phi_{1}(t) \in B_{k}\right\}$ is open and $\phi_{1}(\mathbb{T})=\bigcup_{k=1}^{m} U_{k}$. Define

$$
V_{1}=U_{1}, \quad V_{k}=U_{k} \backslash \bigcup_{i=1}^{k-1} U_{i}, \quad 2 \leq k \leq m .
$$

Then it is clear that $V_{i} \cap V_{j} \neq \emptyset$ if $i \neq j, 1 \leq i, j \leq m$. So we get

$$
\begin{aligned}
& \left\{t \in\left[t_{0}-r, t_{0}+r\right]_{\mathbb{T}}:\left\|\phi\left(t, \phi_{1}(t)\right)\right\| \geq \frac{\varepsilon}{2}\right\} \\
& \quad \subset \bigcup_{k=1}^{m}\left\{t \in V_{k}:\left\|\phi\left(t, \phi_{1}(t)\right)-\phi\left(t, x_{k}\right)\right\|+\left\|\phi\left(t, x_{k}\right)\right\| \geq \varepsilon\right\} \\
& \quad \subset \bigcup_{k=1}^{m}\left(\left\{t \in V_{k}:\left\|\phi\left(t, \phi_{1}(t)\right)-\phi\left(t, x_{k}\right)\right\| \geq \frac{\varepsilon}{2}\right\} \cup\left\{t \in V_{k}:\left\|\phi\left(t, x_{k}\right)\right\| \geq \frac{\varepsilon}{2}\right\}\right) .
\end{aligned}
$$

In view of (3), it follows that

$$
\left\{t \in V_{k}:\left\|\phi\left(t, \phi_{1}(t)\right)-\phi\left(t, x_{k}\right)\right\| \geq \frac{\varepsilon}{2}\right\}=\emptyset, \quad k=1,2, \ldots, m .
$$

Thus we get

$$
\frac{1}{m\left(r, \rho, t_{0}\right)} \mu_{\nabla}\left(M_{r, \varepsilon, t_{0}}\left(\phi\left(t, \phi_{1}(t)\right)\right)\right) \leq \sum_{k=1}^{m} \frac{1}{m\left(r, \rho, t_{0}\right)} \mu_{\nabla}\left(M_{r, \varepsilon, t_{0}}\left(\phi\left(t, x_{k}\right)\right)\right) \text {. }
$$

Since $\phi \in P A A_{0}(\mathbb{T} \times \mathbb{X}, \rho)$ and $\lim _{r \rightarrow \infty} \frac{1}{m\left(r, \rho, t_{0}\right)} \mu_{\nabla}\left(M_{r, \varepsilon, t_{0}}\left(\phi\left(t, x_{k}\right)\right)\right)=0$, it follows that

$$
\lim _{r \rightarrow \infty} \frac{1}{m\left(r, \rho, t_{0}\right)} \mu_{\nabla}\left(M_{r, \varepsilon, t_{0}}\left(\phi\left(t, \phi_{1}(t)\right)\right)\right)=0,
$$

by Lemma 3.2, $\phi\left(\cdot, \phi_{1}(\cdot)\right) \in P A A_{0}(\mathbb{T}, \rho)$. This completes the proof. 
Theorem 3.10 has the following consequence.

Corollary 3.11 Let $f=g+\phi \in W P A P(\mathbb{T}, \rho)$, where $\rho \in U_{B}$. Assume that $f$ and $g$ are Lipschitzian in $x \in \mathbb{X}$ uniformly in $t \in \mathbb{T}$. Then $f(\cdot, h(\cdot)) \in \operatorname{WPAA}(\mathbb{T}, \rho)$ if $h \in \operatorname{WPAA}(\mathbb{T}, \rho)$.

Next, we will show the following two lemmas, which are useful in the proof of our results.

Lemma 3.12 If $\varphi \in P C_{\mathrm{ld}}(\mathbb{T}, \mathbb{X})$ is an almost automorphic function with respect to the sequence $T$ and $\left\{t_{k}\right\} \subset \mathbb{T}$ is equipotentially almost automorphic satisfying $\inf _{i \in \mathbb{Z}} t_{i}^{q}=\theta>0$, $q \in \mathbb{Z}$, then $\left\{\varphi\left(t_{k}\right)\right\}$ is an almost automorphic sequence in $\mathbb{X}$.

Proof Let $t_{i}^{j}=t_{i+j}-t_{i}, i, j \in \mathbb{Z}$. Obviously, from the definition of $\Pi$, it is easy to know that $t_{i}^{j} \in \Pi$. Since $\varphi \in P C_{\mathrm{ld}}(\mathbb{T}, \mathbb{X})$ is an almost automorphic function and $\left\{t_{k}\right\} \subset \mathbb{T}$ is equipotentially almost automorphic, from Definition 3.3 and Definition 3.4, for any sequence $\left\{s_{n}\right\} \subset \mathbb{Z}$, we find that there exists a subsequence $\left\{s_{n}^{\prime}\right\}$ such that

$$
\begin{aligned}
\lim _{n \rightarrow \infty}\left(\varphi\left(t_{k+s_{n}^{\prime}}\right), T-t_{k}^{s_{n}^{\prime}}\right) & =\lim _{n \rightarrow \infty}\left(\varphi\left(t_{k}+t_{k}^{s_{n}^{\prime}}\right), T-t_{k}^{s_{n}^{\prime}}\right)=\left(\varphi^{*}\left(t_{k}\right), T^{*}\right) \\
& =\left(\varphi\left(t_{k}+\gamma_{k}\right), T-\gamma_{k}\right)
\end{aligned}
$$

and

$$
\begin{aligned}
\lim _{n \rightarrow \infty}\left(\varphi^{*}\left(t_{k-s_{n}^{\prime}}\right), T^{*}+t_{k}^{-s_{n}^{\prime}}\right) & =\lim _{n \rightarrow \infty}\left(\varphi\left(t_{k-s_{n}^{\prime}}+\gamma_{k-s_{n}^{\prime}}\right), T-\gamma_{k-s_{n}^{\prime}}+t_{k}^{-s_{n}^{\prime}}\right) \\
& =\left(\varphi\left(t_{k}\right), T\right) .
\end{aligned}
$$

Hence, $\left\{\varphi\left(t_{k}\right)\right\}$ is an almost automorphic sequence in $\mathbb{X}$. This completes the proof.

Lemma 3.13 A necessary and sufficient condition for a bounded sequence $\left\{a_{n}\right\}$ to be in $P A A_{0}(\mathbb{Z}, \rho)$ is that there exists a uniformly continuous function $f \in P A A_{0}(\mathbb{T}, \rho)$ such that $f\left(t_{n}\right)=a_{n}, t_{n} \in \mathbb{T}, n \in \mathbb{Z}, \rho \in U_{B}$.

Proof Necessity. We define a function

$$
f(t)=a_{n}+\left(t-t_{0}-n r\right)\left(a_{n+1}-a_{n}\right), \quad t_{0}+n r \leq t<t_{0}+(n+1) r, t \in \mathbb{T}, n \in \mathbb{Z}, t_{0} \in \mathbb{T},
$$

where $r \in \Pi$. It is obviously uniformly continuous on $\mathbb{T} . f \in P A A_{0}(\mathbb{T}, \rho)$ since

$$
\begin{aligned}
& \frac{1}{m\left(k r, \rho, t_{0}\right)} \int_{t_{0}-k r}^{t_{0}+k r}\|f(s)\| \rho(s) \nabla s \\
& =\frac{1}{m\left(k r, \rho, t_{0}\right)} \sum_{j=-k}^{k-1} \int_{t_{0}+j r}^{t_{0}+(j+1) r}\left\|a_{j}+\left(s-t_{0}-j r\right)\left(a_{j+1}-a_{j}\right)\right\| \rho(s) \nabla s \\
& \leq \frac{1}{m\left(k r, \rho, t_{0}\right)} \underline{\rho} \sum_{j=-k}^{k-1}\left(\left\|a_{j}\right\| \rho\left(t_{j}\right) r+\left\|a_{j+1}-a_{j}\right\| \int_{t_{0}+j r}^{t_{0}+(j+1) r}\left(s-t_{0}-j r\right) \rho(s) \nabla s\right) \\
& \leq \frac{1}{\underline{\rho} m\left(k r, \rho, t_{0}\right)} \sum_{j=-k}^{k-1} r\left\|a_{j}\right\| \rho\left(t_{j}\right)+\frac{\left(\left\|a_{k}\right\|+\left\|a_{-k}\right\|\right) r^{2}}{m\left(k r, \rho, t_{0}\right)} \bar{\rho}
\end{aligned}
$$




$$
\begin{aligned}
& \leq \frac{1}{\underline{\rho} \sum_{t_{j} \in\left[t_{0}-k r, t_{0}+k r\right]_{\mathbb{T}}} v\left(t_{j}\right) \rho\left(t_{j}\right)} \sum_{j=-k}^{k-1} r\left\|f\left(t_{j}\right)\right\| \rho\left(t_{j}\right)+\frac{\left\|a_{k}\right\|+\left\|a_{-k}\right\|}{m\left(k r, \rho, t_{0}\right)} r^{2} \bar{\rho} \\
& =\frac{1}{\underline{\rho} \sum_{j=-k}^{k-1} v\left(t_{j}\right) \rho\left(t_{j}\right)} \sum_{j=-k}^{k-1} r\left\|f\left(t_{j}\right)\right\| \rho\left(t_{j}\right)+\frac{\left\|a_{k}\right\|+\left\|a_{-k}\right\|}{m\left(k r, \rho, t_{0}\right)} r^{2} \bar{\rho} \rightarrow 0 \quad \text { as } k \rightarrow \infty,
\end{aligned}
$$

where $\underline{\rho}=\inf _{t \in \mathbb{T}} \rho(t), \bar{\rho}=\sup _{t \in \mathbb{T}} \rho(t)$.

Sufficiency. Let $0<\varepsilon<1$, there exists $\delta>0$ such that, for $t \in\left(t_{n}-\delta, t_{n}\right)_{\mathbb{T}}, n \in \mathbb{Z}$, we have

$$
\|f(t)\| \rho(t) \geq(1-\varepsilon)\left\|f\left(t_{n}\right)\right\| \rho\left(t_{n}\right), \quad n \in \mathbb{Z} .
$$

Without loss of generality, let $t_{n} \geq 0, t_{-n}<0, n \in \mathbb{Z}$, there exist $r_{n}, r_{-n} \in \Pi \cap \mathbb{R}^{+}$such that $t_{0}+r_{n}=t_{n}, t_{0}-r_{-n}=t_{-n}$. Let $r_{n}^{\prime}=\max \left\{r_{n}, r_{-n}\right\} \in \Pi$. Therefore,

$$
\begin{aligned}
\int_{t_{0}-r_{n}^{\prime}}^{t_{0}+r_{n}^{\prime}}\|f(t)\| \rho(t) \nabla t & \geq \int_{t_{0}-r_{-n}}^{t_{0}+r_{n}}\|f(t)\| \rho(t) \nabla t=\int_{t_{-n}}^{t_{n}}\|f(t)\| \rho(t) \nabla t \\
& \geq \sum_{j=-n+1}^{n} \int_{t_{0}+t_{j-1}}^{t_{0}+t_{j}}\|f(t)\| \rho(t) \nabla t \\
& \geq \sum_{j=-n+1}^{n} \int_{t_{0}+t_{j}-\delta}^{t_{0}+t_{j}}\|f(t)\| \rho(t) \nabla t \\
& \geq \sum_{j=-n+1}^{n} \delta(1-\varepsilon)\left\|f\left(t_{j}\right)\right\| \rho\left(t_{j}\right) \\
& \geq \delta(1-\varepsilon) \sum_{j=-n+1}^{n}\left\|f\left(t_{j}\right)\right\| \rho\left(t_{j}\right),
\end{aligned}
$$

so one can obtain

$$
\frac{1}{m\left(r_{n}^{\prime}, \rho, t_{0}\right)} \int_{t_{0}-r_{n}^{\prime}}^{t_{0}+r_{n}^{\prime}}\|f(t)\| \rho(t) \nabla t \geq \delta(1-\varepsilon) \frac{1}{m\left(r_{n}^{\prime}, \rho, t_{0}\right)} \sum_{j=-n+1}^{n}\left\|f\left(t_{j}\right)\right\| \rho\left(t_{j}\right),
$$

it is easy to see that $r_{n}^{\prime}$ is increasing with respect to $n \in \mathbb{Z}^{+}$, one can find some $n_{0}>n$ such that

$$
m\left(r_{n}^{\prime}, \rho, t_{0}\right)=\int_{t_{0}-r_{n}^{\prime}}^{t_{0}+r_{n}^{\prime}} \rho(s) \nabla s \leq \sum_{t_{j} \in\left[t_{0}-r_{n_{0}}^{\prime}, t_{0}+r_{n_{0}}^{\prime}\right]_{\mathbb{T}}} v\left(t_{j}\right) \rho\left(t_{j}\right)=\sum_{j=-n_{0}+1}^{n_{0}} v\left(t_{j}\right) \rho\left(t_{j}\right),
$$

from (4) and (5), we have

$$
\frac{1}{m\left(r_{n}^{\prime}, \rho, t_{0}\right)} \int_{t_{0}-r_{n}^{\prime}}^{t_{0}+r_{n}^{\prime}}\|f(t)\| \rho(t) \nabla t \geq \delta(1-\varepsilon) \frac{1}{\sum_{j=-n_{0}+1}^{n_{0}} v\left(t_{j}\right) \rho\left(t_{j}\right)} \sum_{j=-n_{0}+1}^{n_{0}}\left\|\phi_{2}\left(t_{j}\right)\right\| \rho\left(t_{j}\right),
$$

noting that $n \rightarrow \infty$ implies $n_{0} \rightarrow \infty$, since $f \in P A A_{0}(\mathbb{T}, \rho)$, it follows from the inequality (6) that $f\left(t_{n}\right)=a_{n} \in P A A_{0}(\mathbb{Z}, \rho)$. This completes the proof.

By Lemma 3.13, we can straightforwardly get the following theorem. 
Theorem 3.14 A necessary and sufficient condition for a bounded sequence $\left\{a_{n}\right\}$ to be in $\operatorname{WPAA}(\mathbb{Z}, \rho)$ is that there exists a uniformly continuous function $f \in W P A A(\mathbb{T}, \rho)$ such that $f\left(t_{n}\right)=a_{n}, t_{n} \in \mathbb{T}, n \in \mathbb{Z}, \rho \in U_{B}$.

Theorem 3.15 Assume that $\rho \in U_{B}$ and the sequence of vector-valued functions $\left\{I_{i}\right\}_{i \in \mathbb{Z}}$ is weighted pseudo almost automorphic, i.e., for any $x \in \Omega,\left\{I_{i}(x), i \in \mathbb{Z}\right\}$ is weighted pseudo almost automorphic sequence. Suppose $\left\{I_{i}(x): i \in \mathbb{Z}, x \in K\right\}$ is bounded for every bounded subset $K \subseteq \Omega, I_{i}(x)$ is uniformly continuous in $x \in \Omega$ uniformly in $i \in \mathbb{Z}$. If $\in W P A A(\mathbb{T}, \rho) \cap$ $U P C(\mathbb{T}, \mathbb{X})$ such that $h(\mathbb{T}) \subset \Omega$, then $I_{i}\left(h\left(t_{i}\right)\right)$ is a weighted pseudo almost automorphic sequence.

Proof Fix $h \in W P A A(\mathbb{T}, \rho) \cap U P C(\mathbb{T}, \mathbb{X})$, first we show $h\left(t_{i}\right)$ is weighted pseudo almost automorphic. Since $h=\phi_{1}+\phi_{2}$, where $\phi_{1} \in A A(\mathbb{T}, \mathbb{X}), \phi_{2} \in P A A_{0}(\mathbb{T}, \rho)$. It follows from Lemma 3.12 that the sequence $\phi_{1}\left(t_{i}\right)$ is almost automorphic. To show that $h\left(t_{i}\right)$ is weighted pseudo almost automorphic, we need to show that $\phi_{2}\left(t_{i}\right) \in P A A_{0}(\mathbb{Z}, \rho)$. By the assumption, $h, \phi_{1} \in U P C(\mathbb{T}, \mathbb{X})$, so is $\phi_{2}$. Let $0<\varepsilon<1$, there exists $\delta>0$ such that, for $t \in\left(t_{i}-\delta, t_{i}\right)_{\mathbb{T}}, i \in \mathbb{Z}$, we have

$$
\left\|\phi_{2}(t)\right\| \rho(t) \geq(1-\varepsilon)\left\|\phi_{2}\left(t_{i}\right)\right\| \rho\left(t_{i}\right), \quad i \in \mathbb{Z} .
$$

Without loss of generality, let $t_{i} \geq 0, t_{-i}<0, i \in \mathbb{Z}$; there exists $r_{i}, r_{-i} \in \Pi \cap \mathbb{R}^{+}$such that $t_{0}+r_{i}=t_{i}, t_{0}-r_{-i}=t_{-i}$. Let $r_{i}^{\prime}=\max \left\{r_{i}, r_{-i}\right\} \in \Pi$. Therefore,

$$
\begin{aligned}
\int_{t_{0}-r_{i}^{\prime}}^{t_{0}+r_{i}^{\prime}}\left\|\phi_{2}(t)\right\| \rho(t) \nabla t & \geq \int_{t_{0}-r_{-i}}^{t_{0}+r_{i}}\left\|\phi_{2}(t)\right\| \rho(t) \nabla t=\int_{t_{-i}}^{t_{i}}\left\|\phi_{2}(t)\right\| \rho(t) \nabla t \\
& \geq \sum_{j=-i+1}^{i} \int_{t_{0}+t_{j-1}}^{t_{0}+t_{j}}\left\|\phi_{2}(t)\right\| \rho(t) \nabla t \\
& \geq \sum_{j=-i+1}^{i} \int_{t_{0}+t_{j}-\delta}^{t_{0}+t_{j}}\left\|\phi_{2}(t)\right\| \rho(t) \nabla t \\
& \geq \sum_{j=-i+1}^{i} \delta(1-\varepsilon)\left\|\phi_{2}\left(t_{j}\right)\right\| \rho\left(t_{j}\right) \\
& \geq \delta(1-\varepsilon) \sum_{j=-i+1}^{i}\left\|\phi_{2}\left(t_{j}\right)\right\| \rho\left(t_{j}\right),
\end{aligned}
$$

so one can obtain

$$
\frac{1}{m\left(r_{i}^{\prime}, \rho, t_{0}\right)} \int_{t_{0}-r_{i}^{\prime}}^{t_{0}+r_{i}^{\prime}}\left\|\phi_{2}(t)\right\| \rho(t) \nabla t \geq \delta(1-\varepsilon) \frac{1}{m\left(r_{i}^{\prime}, \rho, t_{0}\right)} \sum_{j=-i+1}^{i}\left\|\phi_{2}\left(t_{j}\right)\right\| \rho\left(t_{j}\right),
$$

it is easy to see that $r_{i}^{\prime}$ is increasing with respect to $i \in \mathbb{Z}^{+}$, and one can find some $i_{0}>i$ such that

$$
m\left(r_{i}^{\prime}, \rho, t_{0}\right)=\int_{t_{0}-r_{i}^{\prime}}^{t_{0}+r_{i}^{\prime}} \rho(s) \nabla s \leq \sum_{t_{j} \in\left[t_{0}-r_{i_{0}}^{\prime}, t_{0}+r_{i_{0}}^{\prime}\right]_{\mathbb{T}}} v\left(t_{j}\right) \rho\left(t_{j}\right)=\sum_{j=-i_{0}+1}^{i_{0}} v\left(t_{j}\right) \rho\left(t_{j}\right),
$$


from (7) and (8), we have

$$
\frac{1}{m\left(r_{i}^{\prime}, \rho, t_{0}\right)} \int_{t_{0}-r_{i}^{\prime}}^{t_{0}+r_{i}^{\prime}}\left\|\phi_{2}(t)\right\| \rho(t) \nabla t \geq \delta(1-\varepsilon) \frac{1}{\sum_{j=-i_{0}+1}^{i_{0}} v\left(t_{j}\right) \rho\left(t_{j}\right)} \sum_{j=-i_{0}+1}^{i_{0}}\left\|\phi_{2}\left(t_{j}\right)\right\| \rho\left(t_{j}\right),
$$

noting that $i \rightarrow \infty$ implies $i_{0} \rightarrow \infty$, since $\phi_{2} \in P A A_{0}(\mathbb{T}, \rho)$, it follows from the inequality (9) that $\phi_{2}\left(t_{i}\right) \in P A A_{0}(\mathbb{Z}, \rho)$. Hence, $h\left(t_{i}\right)$ is weighted pseudo almost automorphic.

Now, we show that $I_{i}\left(\phi\left(t_{i}\right)\right)$ is weighted pseudo almost automorphic. Let

$$
\begin{array}{ll}
I(t, x)=I_{n}(x)+\left(t-t_{0}-n r\right)\left[I_{n+1}(x)-I_{n}(x)\right], & t_{0}+n r \leq t<t_{0}+(n+1) r, n \in \mathbb{Z}, r \in \Pi, \\
\Phi_{0}(t)=h\left(t_{n}\right)+\left(t-t_{0}-n r\right)\left[h\left(t_{n+1}\right)-h\left(t_{n}\right)\right], & t_{0}+n r \leq t<t_{0}+(n+1) r, n \in \mathbb{Z}, r \in \Pi .
\end{array}
$$

Since $I_{n}, h\left(t_{n}\right)$ both are pseudo almost automorphic, by Lemma 3.13 and Theorem 3.14, we know that $I \in W P A A(\mathbb{T} \times \Omega, \rho), \Phi_{0} \in W P A A(\mathbb{T}, \rho)$. For every $t \in \mathbb{T}$, there exists a number $n \in \mathbb{Z}$ such that $\left|t-t_{0}-n r\right| \leq r$,

$$
\begin{aligned}
\|I(t, x)\| & \leq\left\|I_{n}(x)\right\|+\left|t-t_{0}-n r\right|\left[\left\|I_{n+1}(x)\right\|+\left\|I_{n}(x)\right\|\right] \\
& \leq(1+r)\left\|I_{n}(x)\right\|+r\left\|I_{n+1}(x)\right\| .
\end{aligned}
$$

Since $\left\{I_{n}(x): n \in \mathbb{Z}, x \in K\right\}$ is bounded for every bounded set $K \subseteq \Omega,\{I(t, x): t \in \mathbb{T}, x \in K\}$ is bounded for every bounded set $K \subseteq \Omega$. For every $x_{1}, x_{2} \in \Omega$, we have

$$
\begin{aligned}
\left\|I\left(t, x_{1}\right)-I\left(t, x_{2}\right)\right\| \leq & \left\|I_{n}\left(x_{1}\right)-I_{n}\left(x_{2}\right)\right\|+\left|t-t_{0}-n r\right|\left[\left\|I_{n+1}\left(x_{1}\right)-I_{n+1}\left(x_{2}\right)\right\|\right. \\
& \left.+\left\|I_{n}\left(x_{1}\right)-I_{n}\left(x_{2}\right)\right\|\right] \\
\leq & (1+r)\left\|I_{n}\left(x_{1}\right)-I_{n}\left(x_{2}\right)\right\|+r\left\|I_{n+1}\left(x_{1}\right)-I_{n+1}\left(x_{2}\right)\right\| .
\end{aligned}
$$

Noting that $I_{i}(x)$ is uniformly continuous in $x \in \Omega$ uniformly in $i \in \mathbb{Z}$, we then find that $I(t, x)$ is uniformly in $x \in \Omega$ uniformly in $t \in \mathbb{T}$. Then by Theorem 3.10, $I\left(\cdot, \Phi_{0}(\cdot)\right) \in$ $\operatorname{WPAA}(\mathbb{T}, \mathbb{X})$. Again, using Lemma 3.13 and Theorem 3.14, we find that $I\left(i, \Phi_{0}(i)\right)$ is a weighted pseudo almost automorphic sequence, that is, $I_{i}\left(h\left(t_{i}\right)\right)$ is weighted pseudo almost automorphic. This completes the proof.

From Theorem 3.15, one can easily get the following corollary.

Corollary 3.16 Assume the sequence of vector-valued functions $\left\{I_{i}\right\}_{i \in \mathbb{Z}}$ is weighted pseudo almost automorphic, $\rho \in U_{B}$, if there is a number $L>0$ such that

$$
\left\|I_{i}(x)-I_{i}(y)\right\| \leq L\|x-y\|
$$

for all $x, y \in \Omega, i \in \mathbb{Z}$, if $h \in W P A A(\mathbb{T}, \rho) \cap U P C(\mathbb{T}, \rho)$ such that $h(\mathbb{T}) \subset \Omega$, then $I_{i}\left(h\left(t_{i}\right)\right)$ is a weighted pseudo almost automorphic sequence.

\section{Weighted piecewise pseudo almost automorphic mild solutions to abstract impulsive $\nabla$-dynamic equations}

In this section, we investigate the existence and exponential stability of a weighted piecewise pseudo almost automorphic mild solution to Eq. (1). Before starting our investigation, we will show a lemma which will be used in our proofs. 
Lemma 4.1 Let $\ominus_{v} \omega \in \mathcal{R}_{v}^{+}$, for all $t \in \mathbb{T}, \alpha \in \Pi$, there exist constants $\beta_{1}, \beta_{2}>0$ such that

$$
\beta_{1} v(t) \leq v(t+\alpha) \leq \beta_{2} v(t)
$$

Then there exist positive constants $K^{*}$ and $\omega^{*}$ such that

$$
\hat{e}_{\ominus_{\nu} \omega}(t+\alpha, s+\alpha) \leq K^{*} \hat{e}_{\ominus v} \omega^{*}(t, s), \quad t \geq s .
$$

Proof Obviously, if $v=0, \mathbb{T}=\mathbb{R}$, the result holds. Assume that $v \not \equiv 0$. Since $\ominus_{\nu} \omega \in \mathcal{R}_{v}^{+}$, one has

$$
\begin{aligned}
e_{\ominus_{\nu} \omega}(t+\alpha, s+\alpha) & =\exp \left\{-\int_{s+\alpha}^{t+\alpha} \frac{1}{v(\tau)} \ln \frac{1}{1-v(\tau) \omega} \nabla \tau\right\} \\
& =\exp \left\{-\int_{s}^{t} \frac{1}{v(\tau+\alpha)} \ln \frac{1}{1-v(\tau+\alpha) \omega} \nabla \tau\right\} .
\end{aligned}
$$

Since $\mathbb{T}$ is an almost periodic time scale, $\mu$ is bounded. Hence, by the inequality (10), we can obtain

$$
\begin{aligned}
\hat{e}_{\ominus_{\nu} \omega}(t+\alpha, s+\alpha) & \leq \exp \left\{-\int_{s}^{t} \frac{1}{\beta_{2} v(\tau)} \ln \frac{1}{1-\beta_{1} v(\tau) \omega} \nabla \tau\right\} \\
& =\left\{\exp \left\{-\int_{s}^{t} \frac{\ln \left(1-v(\tau)\left(\ominus_{\nu} \beta_{1} \omega\right)\right)}{v(\tau)}\right\}\right\}^{\frac{1}{\beta_{2}}} .
\end{aligned}
$$

Therefore, there exists a positive constant $K^{*}>0$ such that

$$
\hat{e}_{\ominus_{v} \omega}(t+\alpha, s+\alpha)=\left[\hat{e}_{\ominus v} \beta_{1} \omega(t, s)\right]^{\frac{1}{\beta_{2}}} \leq K^{*} \hat{e}_{\ominus_{\nu} \omega^{*}}(t, s),
$$

where $\omega^{*}=\beta_{1} \omega$. This completes the proof.

Remark 4.2 It is easy to see that if $\mathbb{T}$ is almost periodic, then $\mu(t)$ is bounded, so there exist a sufficiently small constant $\beta_{1}>0$ and a sufficiently large constant $\beta_{2}>0$ such that (10) is valid. Therefore, Lemma 4.1 holds when $\mathbb{T}$ is an almost periodic time scale.

Let $\mathbb{T}$ be an almost periodic time scale, and consider the impulsive $\nabla$-dynamic equation

$$
x^{\nabla}=A(t) x^{\varrho}, \quad t \in \mathbb{T},
$$

where $A: \mathbb{T} \rightarrow B(\mathbb{X})$ is a linear operator in the Banach space $\mathbb{X}$. We denote by $B(\mathbb{X}, \mathbb{Y})$ the Banach space of all bounded linear operators from $\mathbb{X}$ to $\mathbb{Y}$. This is simply denoted as $B(\mathbb{X})$ when $\mathbb{X}=\mathbb{Y}$.

Definition 4.1 $T(t, s): \mathbb{T} \times \mathbb{T} \rightarrow B(\mathbb{X})$ is called the linear evolution operator associated to (11) if $T(t, s)$ satisfies the following conditions:

(1) $T(s, s)=$ Id, where Id denotes the identity operator in $\mathbb{X}$;

(2) $T(t, s) T(s, r)=T(t, r)$;

(3) the mapping $(t, s) \rightarrow T(t, s) x$ is continuous for any fixed $x \in \mathbb{X}$. 
Definition 4.2 An evolution system $T(t, s)$ is called exponentially stable if there exist $K_{0} \geq$ 1 and $\omega>0$ such that

$$
\|T(t, s)\|_{B(\mathbb{X})} \leq K_{0} \hat{e}_{\ominus_{\nu} \omega}(t, s), \quad t \geq s
$$

Definition 4.3 A function $x: \mathbb{T} \rightarrow \mathbb{X}$ is called a mild solution of Eq. (1) if, for any $t \in \mathbb{T}$, $t>c, c \neq t_{i}, i \in \mathbb{Z}$, one has

$$
x(t)=T(t, c) x(c)+\int_{c}^{t} T(t, s) f(s, x(s)) \nabla s+\sum_{c<t_{i}<t} T\left(t, t_{i}\right) I_{i}\left(x\left(t_{i}\right)\right) .
$$

In fact, using the semigroup theory, we can easily see that

$$
x(t)=T(t, c) x(c)+\int_{c}^{t} T(t, s) f(s, x(s)) \nabla s, \quad t>c,
$$

is a mild solution to

$$
x^{\nabla}=A(t) x^{\varrho}+f(t, x(t)) .
$$

For any $c \in \mathbb{T}$, we can find $i \in \mathbb{Z}, t_{i-1}<c \leq t_{i}$, for $t \in\left(c, t_{i}\right]_{\mathbb{T}}$,

$$
x(t)=T(t, c) x(c)+\int_{c}^{t} T(t, s) f(s, x(s)) \nabla s,
$$

by using $x\left(t_{i}^{+}\right)-x\left(t_{i}^{-}\right)=I_{i}\left(x\left(t_{i}\right)\right)$, we have

$$
x\left(t_{i}^{+}\right)=T\left(t_{i}, c\right) x(c)+\int_{c}^{t_{i}} T\left(t_{i}, s\right) f(s, x(s)) \nabla s+I_{i}\left(x\left(t_{i}\right)\right),
$$

then we have

$$
\begin{aligned}
x(t)= & T\left(t, t_{i}\right) x\left(t_{i}^{+}\right)+\int_{t_{i}}^{t} T(t, s) f(s, u(s)) \nabla s \\
= & T\left(t, t_{i}\right)\left[T\left(t_{i}, c\right) x(c)+\int_{c}^{t_{i}} T\left(t_{i}, s\right) f(s, x(s)) \nabla s+I_{i}\left(x\left(t_{i}\right)\right)\right] \\
& +\int_{t_{i}}^{t} T(t, s) f(s, x(s)) \nabla s \\
= & T(t, c) x(c)+\int_{c}^{t_{i}} T(t, s) f(s, x(s)) \nabla s+T\left(t, t_{i}\right) I_{i}\left(x\left(t_{i}\right)\right) \\
& +\int_{t_{i}}^{t} T(t, s) f(s, x(s)) \nabla s \\
= & T(t, c) x(c)+\int_{c}^{t} T(t, s) f(s, x(s)) \nabla s+T\left(t, t_{i}\right) I_{i}\left(x\left(t_{i}\right)\right) .
\end{aligned}
$$

Repeating this procedure, we get

$$
x(t)=T(t, c) x(c)+\int_{c}^{t} T(t, s) f(s, x(s)) \nabla s+\sum_{c<t_{i}<t} T\left(t, t_{i}\right) I_{i}\left(x\left(t_{i}\right)\right) .
$$


In the following, consider the abstract differential system (1) with the following assumptions:

$\left(\mathrm{H}_{1}\right)$ The family $\{A(t): t \in \mathbb{T}\}$ of operators in $\mathbb{X}$ generates an exponentially stable evolution system $\{T(t, s): t \geq s\}$, i.e., there exist $K_{0}>1$ and $\omega>0$ such that

$$
\|T(t, s)\|_{B(\mathbb{X})} \leq K_{0} e_{\ominus \omega}(t, s), \quad t \geq s
$$

and for any sequence $\left\{s_{n}\right\} \subset \Pi$, there exists a subsequence $\left\{s_{n}^{\prime}\right\} \subset\left\{s_{n}\right\}$ such that

$$
\lim _{n \rightarrow \infty} T\left(t+s_{n}^{\prime}, s+s_{n}^{\prime}\right)=T^{*}(t, s) \text { is well defined for each } t, s \in \mathbb{T}, t \geq s
$$

$\left(\mathrm{H}_{2}\right) f=g+\phi \in \operatorname{WPAP}(\mathbb{T}, \rho)$, where $\rho \in U_{\infty}$ and $f(t, \cdot)$ is uniformly continuous in each bounded subset of $\Omega$ uniformly in $t \in \mathbb{T} ; I_{i}$ is a weighted pseudo almost periodic sequence, $I_{i}(x)$ is uniformly continuous in $x \in \Omega$ uniformly in $i \in \mathbb{Z}$, $\inf _{i \in \mathbb{Z}} t_{i}^{1}=\theta>0$.

To investigate the existence and uniqueness of a weighted piecewise pseudo almost automorphic mild solution to Eq. (1), we need the following lemma.

Lemma 4.3 Let $v \in A A(\mathbb{T}, \mathbb{X}), v \in A A\left(\mathbb{T}, \mathbb{R}^{+}\right), \omega \in \mathcal{R}_{v}^{+}$and $\left(\mathrm{H}_{1}\right)-\left(\mathrm{H}_{2}\right)$ are satisfied. If $u$ : $\mathbb{T} \rightarrow \mathbb{X}$ is defined by

$$
u_{0}(t)=\int_{-\infty}^{t} T(t, s) v(s) \nabla s+\sum_{t_{i}<t} T\left(t, t_{i}\right) I_{i}\left(v\left(t_{i}\right)\right), \quad t \geq s
$$

then $u_{0}(\cdot) \in A A(\mathbb{T}, \mathbb{X})$.

Proof Let $\left\{s_{n}\right\}_{n=1}^{\infty} \subset \Pi$. Since $v$ is almost automorphic, there exists a subsequence $\left\{\tau_{n}\right\}_{n=1}^{\infty} \subset$ $\left\{s_{n}\right\}_{n=1}^{\infty}$ such that $h(t):=\lim _{n \rightarrow \infty} v\left(t+\tau_{n}\right)$ is well defined for each $t \in \mathbb{T}$.

Now, we consider

$$
\begin{aligned}
u\left(t+\tau_{n}\right) & =\int_{-\infty}^{t+\tau_{n}} T\left(t+\tau_{n}, s\right) v(s) \nabla s=\int_{-\infty}^{t} T\left(t+\tau_{n}, s+\tau_{n}\right) v\left(s+\tau_{n}\right) \nabla s \\
& =\int_{-\infty}^{t} T\left(t+\tau_{n}, s+\tau_{n}\right) v_{n}(s) \nabla s,
\end{aligned}
$$

where $v_{n}(s)=v\left(s+\tau_{n}\right), n=1,2, \ldots$.

Since $\omega \in \mathcal{R}_{v}^{+}$, one can choose sufficiently small constant $\beta_{1}>0$ such that $\omega^{*}=\beta_{1} \omega$ is $v$-positive regressive. Further, noting that $\hat{e}_{\ominus_{v} \omega^{*}}(t, s)\left(1-v(s) \omega^{*}\right)=\hat{e}_{\ominus_{v} \omega^{*}}(t, \varrho(s))$, by $\left(\mathrm{H}_{1}\right)$ and Lemma 4.1, we have

$$
\begin{aligned}
\left\|u\left(t+\tau_{n}\right)\right\| & \leq \int_{-\infty}^{t}\left\|T\left(t+\tau_{n}, s+\tau_{n}\right) v_{n}(s)\right\| \nabla s \\
& \leq \int_{-\infty}^{t} K_{0} \hat{e}_{\ominus_{\nu} \omega}\left(t+\tau_{n}, s+\tau_{n}\right)\left\|v_{n}(s)\right\| \nabla s \\
& \leq K_{0} K^{*} \int_{-\infty}^{t} \hat{e}_{\ominus_{\nu} \omega^{*}}(t, s)\left\|v_{n}(s)\right\| \nabla s \\
& \leq \frac{1}{1-\bar{v} \omega^{*}} K_{0} K^{*}\|v\| \int_{-\infty}^{t} \hat{e}_{\ominus \nu} \omega^{*}(t, \varrho(s)) \nabla s
\end{aligned}
$$




$$
\begin{aligned}
& =\frac{K_{0} K^{*}\|v\|}{\left(1-\bar{v} \omega^{*}\right) \ominus_{\nu} \omega^{*}}\left[\hat{e}_{\ominus_{\nu} \omega^{*}}(t,-\infty)-\hat{e}_{\ominus_{\nu} \omega^{*}}(t, t)\right] \\
& \leq \frac{K_{0} K^{*}\left(1-\underline{v} \omega^{*}\right)\|v\|}{\left(1-\bar{v} \omega^{*}\right) \omega^{*}}
\end{aligned}
$$

where $\bar{v}=\sup _{t \in \mathbb{T}} v(t), \underline{v}=\inf _{t \in \mathbb{T}} v$.

Therefore, by the condition $\left(\mathrm{H}_{1}\right)$, we have

$$
T\left(t+\tau_{n}, s+\tau_{n}\right) \rightarrow T^{*}(t, s), \quad n \rightarrow \infty .
$$

Furthermore, it is easy to see that $v_{n}(s) \rightarrow h(s)$ as $n \rightarrow \infty, \forall s \in \mathbb{T}$ and for any $t \geq s$, by Lebesgue's dominated convergence theorem, we get

$$
\lim _{n \rightarrow \infty} u\left(t+\tau_{n}\right)=\int_{-\infty}^{t} T^{*}(t, s) h(s) \nabla s .
$$

Moreover, we consider

$$
\begin{aligned}
u^{\prime}\left(t+\tau_{n}\right) & =\sum_{t_{i}<t+\tau_{n}} T\left(t+\tau_{n}, t_{i}\right) I_{i}\left(v_{i}\left(t_{i}\right)\right)=\sum_{t_{i}<t} T\left(t+\tau_{n}, t_{i}+\tau_{n}\right) I_{i}\left(v\left(t_{i}+\tau_{n}\right)\right) \\
& =\sum_{t_{i}<t} T\left(t+\tau_{n}, t_{i}+\tau_{n}\right) I_{i}\left(v_{i n}\right),
\end{aligned}
$$

where $v\left(t_{i}+\tau_{n}\right):=v_{i n}$. By Lemma 4.1, we can get

$$
\begin{aligned}
\left\|u^{\prime}\left(t+\tau_{n}\right)\right\| & =\left\|\sum_{t_{i}<t+\tau_{n}} T\left(t+\tau_{n}, t_{i}\right) I_{i}\left(v_{i}\left(t_{i}\right)\right)\right\|=\left\|\sum_{t_{i}<t} T\left(t+\tau_{n}, t_{i}+\tau_{n}\right) I_{i}\left(v_{i n}\right)\right\| \\
& \leq I K_{0} \sum_{t_{i}<t} \hat{e}_{\ominus_{\nu} \omega}\left(t+\tau_{n}, t_{i}+\tau_{n}\right) \\
& \leq I K_{0} K^{*} \sum_{t_{i}<t} \hat{e}_{\ominus_{\nu} \omega^{*}}\left(t, t_{i}\right) \leq \frac{I K_{0} K^{*}}{1-\hat{e}_{\ominus_{\nu} \omega^{*}}(\theta, 0)}
\end{aligned}
$$

Since $v \in A A(\mathbb{T}, \mathbb{X}), v_{\text {in }} \rightarrow h\left(t_{i}\right), n \rightarrow \infty, \forall i \in \mathbb{Z}$. Hence, for any $t>t_{i}, i \in \mathbb{Z}$, by Lebesgue's dominated convergence theorem, we get

$$
\lim _{n \rightarrow \infty} u^{\prime}\left(t+\tau_{n}\right)=\sum_{t_{i}<t} T^{*}\left(t, t_{i}\right) I_{i}\left(h\left(t_{i}\right)\right) .
$$

So we have

$$
\lim _{n \rightarrow \infty} u_{0}\left(t+\tau_{n}\right)=\lim _{n \rightarrow \infty} u\left(t+\tau_{n}\right)+\lim _{n \rightarrow \infty} u^{\prime}\left(t+\tau_{n}\right)
$$

is well defined for each $t \in \mathbb{T}$. Therefore, $u_{0}(\cdot) \in A A(\mathbb{T}, \mathbb{X})$. This completes the proof.

Theorem 4.4 Let $f(\cdot, \vartheta(\cdot)) \in \operatorname{WPAA}(\mathbb{T}, \rho)$, where $\vartheta \in \operatorname{WPAA}(\mathbb{T}, \rho)$ and $\{T(t, s), t \geq s\}$ is exponentially stable, $\rho \in U_{B}$. Then

$$
F(\cdot):=\int_{-\infty}^{(\cdot)} T(\cdot, s) f(s, \vartheta(s)) \nabla s+\sum_{t_{i}<\cdot} T\left(\cdot, t_{i}\right) I_{i}\left(\vartheta\left(t_{i}\right)\right) \in \operatorname{WPAA}(\mathbb{T}, \rho) .
$$


Proof Fix $\vartheta \in \operatorname{WPAA}(\mathbb{T}, \mathbb{X})$, then we have $f(\cdot, \vartheta(\cdot))=\phi_{1}(\cdot)+\phi_{2}(\cdot)$, where $\phi_{1} \in A A(\mathbb{T}, \mathbb{X})$, $\phi_{2} \in P A A_{0}(\mathbb{T}, \mathbb{X})$, so

$$
\int_{-\infty}^{t} T(t, s) f(s, \vartheta(s)) \nabla s=\int_{-\infty}^{t} T(t, s) \phi_{1}(s) \nabla s+\int_{-\infty}^{t} T(t, s) \phi_{2}(s) \nabla s:=I_{1}(t)+I_{2}(t)
$$

and

$$
\sum_{t_{i}<t} T\left(t, t_{i}\right) I_{i}\left(\vartheta\left(t_{i}\right)\right)=\sum_{t_{i}<t} T\left(t, t_{i}\right) \beta_{i}+\sum_{t_{i}<t} T\left(t, t_{i}\right) \gamma_{i}:=\Upsilon_{1}(t)+\Upsilon_{2}(t)
$$

By Lemma 4.3, we can easily see that $I_{1}, \Upsilon_{1} \in A A(\mathbb{T}, \mathbb{X})$.

Moreover, we have

$$
\begin{aligned}
\frac{1}{m\left(r, \rho, t_{0}\right)} \int_{t_{0}-r}^{t_{0}+r}\left\|I_{2}(t)\right\| \nabla t \\
=\frac{1}{m\left(r, \rho, t_{0}\right)} \int_{t_{0}-r}^{t_{0}+r}\left\|\int_{-\infty}^{t} T(t, s) \phi_{2}(s) \nabla s\right\| \nabla t \\
\leq \frac{1}{m\left(r, \rho, t_{0}\right)} \int_{t_{0}-r}^{t_{0}+r} \nabla t \int_{-\infty}^{t} K_{0} \hat{e}_{\ominus_{\nu} \omega}(t, s)\left\|\phi_{2}(s)\right\| \nabla s \\
=\frac{1}{m\left(r, \rho, t_{0}\right)} \int_{t_{0}-r}^{t_{0}+r} \nabla t\left(\int_{-\infty}^{t_{0}-r} K_{0} \hat{e}_{\ominus_{\nu} \omega}(t, s)\left\|\phi_{2}(s)\right\| \nabla s\right. \\
\left.\quad+\int_{t_{0}-r}^{t} K_{0} \hat{e}_{\ominus_{\nu} \omega}(t, s)\left\|\phi_{2}(s)\right\| \nabla s\right) \\
=\frac{1}{m\left(r, \rho, t_{0}\right)} \int_{-\infty}^{t_{0}-r}\left\|\phi_{2}(s)\right\| \nabla s \int_{t_{0}-r}^{t_{0}+r} K_{0} \hat{e}_{\ominus_{\nu} \omega}(t, s) \nabla s \\
\quad+\frac{1}{m\left(r, \rho, t_{0}\right)} \int_{t_{0}-r}^{t_{0}+r}\left\|\phi_{2}(s)\right\| \nabla s \int_{s}^{t_{0}+r} K_{0} \hat{e}_{\ominus_{\nu} \omega}(t, s) \nabla t \\
:=
\end{aligned}
$$

Then

$$
\begin{aligned}
I_{1}^{0}= & \frac{1}{m\left(r, \rho, t_{0}\right)} \int_{-\infty}^{t_{0}-r}\left\|\phi_{2}(s)\right\| \nabla s \int_{t_{0}-r}^{t_{0}+r} K_{0} \hat{e}_{\ominus_{\nu} \omega}(t, s) \nabla t \\
= & \frac{1}{m\left(r, \rho, t_{0}\right)} \int_{-\infty}^{t_{0}-r}\left\|\phi_{2}(s)\right\| \nabla s \int_{t_{0}-r}^{t_{0}+r} \frac{K_{0}}{1-v\left(\ominus_{\nu} \omega\right)} \hat{e}_{\ominus_{\nu} \omega}(\varrho(t), s) \nabla t \\
\leq & \frac{1}{m\left(r, \rho, t_{0}\right)} K_{0}(1-\underline{v} \omega) \int_{-\infty}^{t_{0}-r}\left\|\phi_{2}(s)\right\| \nabla s \int_{t_{0}-r}^{t_{0}+r} \hat{e}_{\omega}(s, \varrho(t)) \nabla t \\
= & \frac{1}{m\left(r, \rho, t_{0}\right)} \frac{K_{0}(1-\underline{v} \omega)}{\omega} \int_{-\infty}^{t_{0}-r}\left\|\phi_{2}(s)\right\|\left[\hat{e}_{\omega}\left(s, t_{0}-r\right)-\hat{e}_{\omega}\left(s, t_{0}+r\right)\right] \nabla s \\
\leq & \frac{1}{m\left(r, \rho, t_{0}\right)} \frac{K_{0}(1-\underline{v} \omega)}{\omega}\left\|\phi_{2}\right\|\left(\int_{-\infty}^{t_{0}-r} \hat{e}_{\ominus_{\nu} \omega}\left(t_{0}-r, s\right) \nabla s-\int_{-\infty}^{t_{0}-r} \hat{e}_{\ominus v}\left(t_{0}+r, s\right) \nabla s\right) \\
= & \frac{1}{m\left(r, \rho, t_{0}\right)} \frac{K_{0}(1-\underline{v} \omega)^{2}}{\omega} \frac{1}{\ominus_{\nu} \omega}\left(\hat{e}_{\ominus_{\nu} \omega}\left(t_{0}-r,-\infty\right)-\hat{e}_{\ominus_{\nu} \omega}\left(t_{0}-r, t_{0}-r\right)\right. \\
& \left.-\hat{e}_{\ominus_{\nu} \omega}\left(t_{0}+r,-\infty\right)+\hat{e}_{\ominus_{\nu} \omega}\left(t_{0}+r, t_{0}-r\right)\right) \rightarrow 0 \quad \text { as } r \rightarrow \infty
\end{aligned}
$$


and

$$
\begin{aligned}
I_{2}^{0} & =\frac{1}{m\left(r, \rho, t_{0}\right)} \int_{t_{0}-r}^{t_{0}+r}\left\|\phi_{2}(s)\right\| \nabla s \int_{s}^{t_{0}+r} K_{0} \hat{e}_{\ominus_{\nu} \omega}(t, s) \nabla t \\
& =\frac{1}{m\left(r, \rho, t_{0}\right)} \int_{t_{0}-r}^{t_{0}+r}\left\|\phi_{2}(s)\right\| \nabla s \int_{s}^{t_{0}+r} \frac{1}{1-v\left(\ominus_{\nu} \omega\right)} \hat{e}_{\ominus_{\nu} \omega}(\varrho(t), s) \nabla t \\
& \leq \frac{1}{m\left(r, \rho, t_{0}\right)} K_{0}(1-\underline{v} \omega) \int_{t_{0}-r}^{t_{0}+r}\left\|\phi_{2}(s)\right\| \nabla s \int_{s}^{t_{0}+r} \hat{e}_{\omega}(s, \varrho(t)) \nabla t \\
& =\frac{1}{m\left(r, \rho, t_{0}\right)} \frac{K_{0}(1-\underline{v} \omega)}{\omega} \int_{t_{0}-r}^{t_{0}+r}\left\|\phi_{2}(s)\right\|\left[\hat{e}_{\omega}(s, s)-\hat{e}_{\omega}\left(s, t_{0}+r\right)\right] \nabla s \\
& \leq \frac{1}{m\left(r, \rho, t_{0}\right)} \frac{K_{0}(1-\underline{v} \omega)}{\omega} \int_{t_{0}-r}^{t_{0}+r}\left\|\phi_{2}(s)\right\| \nabla s .
\end{aligned}
$$

Since $\phi_{2} \in P A A_{0}(\mathbb{T}, \rho)$, we have $\lim _{r \rightarrow \infty} \frac{1}{m\left(r, \rho, t_{0}\right)} \int_{t_{0}-r}^{t_{0}+r}\left\|\phi_{2}(s)\right\| \nabla s=0$. Hence, $\lim _{r \rightarrow \infty} I_{2}^{0}=0$. It remains to show $\Upsilon_{2} \in P A A_{0}(\mathbb{T}, \rho)$. For any $r>0$, there exist $i(r), j(r)$ such that

$$
t_{i(r)-1}<t_{0}-r \leq t_{i(r)}<\cdots<t_{j(r)} \leq t_{0}+r<t_{j(r)+1} .
$$

Since $\gamma_{i} \in P A A_{0}(\mathbb{Z}, \rho), M_{\gamma_{i}}=\sup _{i \in \mathbb{Z}}\left\|\gamma_{i}\right\|<\infty$, and noting that, for $a \in \mathbb{T}, \hat{e}_{\ominus_{\nu} \omega}(t, a)=(1-$ $\nu(t) \omega) \hat{e}_{\omega}(a, \varrho(t))$, we have

$$
\begin{aligned}
& \frac{1}{m\left(r, \rho, t_{0}\right)} \int_{t_{0}-r}^{t_{0}+r}\left\|\Upsilon_{2}(t)\right\| \nabla t \\
& =\frac{1}{m\left(r, \rho, t_{0}\right)} \int_{t_{0}-r}^{t_{0}+r}\left\|\sum_{t_{i}<t} T\left(t, t_{i}\right) \gamma_{i}\right\| \nabla t \\
& \leq \frac{1}{m\left(r, \rho, t_{0}\right)} \int_{t_{0}-r}^{t_{0}+r} \sum_{t_{i}<t} K_{0} \hat{e}_{\ominus_{\nu} \omega}\left(t, t_{i}\right)\left\|\gamma_{i}\right\| \nabla t \\
& \leq \frac{1}{m\left(r, \rho, t_{0}\right)} \sum_{t_{i}<t_{0}-r} K_{0} \hat{e}_{\ominus v \omega}\left(t_{0}-r, t_{i}\right)\left\|\gamma_{i}\right\| \\
& \times \int_{t_{0}-r}^{t_{0}+r} \hat{e}_{\ominus_{\nu} \omega}\left(t, t_{0}-r\right) \nabla t \\
& +\frac{1}{m\left(r, \rho, t_{0}\right)} \sum_{t_{0}-r<t_{i}<t_{0}+r}\left\|\gamma_{i}\right\| \int_{t_{0}-r}^{t_{0}+r} K_{0} \hat{e}_{\ominus_{v} \omega}\left(t, t_{i}\right) \nabla t \\
& \leq \frac{1}{m\left(r, \rho, t_{0}\right)} \sum_{t_{i}<t_{0}-r} \frac{K_{0}(1-\underline{v} \omega)^{2}}{\omega} M_{\gamma_{i}} \hat{e}_{\ominus v \omega}\left(t_{0}-r, t_{i}\right) \\
& +\frac{1}{m\left(r, \rho, t_{0}\right)} \sum_{t_{0}-r<t_{i}<t_{0}+r} \frac{K_{0}(1-\underline{v} \omega)^{2}}{\omega}\left\|\gamma_{i}\right\| \\
& \leq \frac{1}{m\left(r, \rho, t_{0}\right)} \frac{K_{0} M_{\gamma_{i}}(1-\underline{v} \omega)^{2}}{\omega} \frac{1}{1-\hat{e}_{\ominus_{\nu} \omega}(\theta, 0)} \\
& +\frac{K_{0}(1-\underline{v} \omega)^{2}}{\omega} \frac{1}{m\left(r, \rho, t_{0}\right)} \sum_{k=i(r)}^{j(r)}\left\|\gamma_{k}\right\| .
\end{aligned}
$$


Since $\gamma_{i} \in P A A_{0}(\mathbb{Z}, \rho)$, for $r \rightarrow \infty, m(r, \rho) \rightarrow \infty$, we have

$$
\lim _{r \rightarrow \infty} \frac{1}{m\left(r, \rho, t_{0}\right)} \sum_{k=i(r)}^{j(r)}\left\|\gamma_{k}\right\|=\lim _{r \rightarrow \infty} \frac{1}{\sum_{k=i(r)}^{j(r)} \rho\left(t_{k}\right) v\left(t_{k}\right)} \sum_{k=i(r)}^{j(r)}\left\|\gamma_{k}\right\|=0 .
$$

Clearly, as $r \rightarrow \infty$, one has

$$
\frac{1}{m\left(r, \rho, t_{0}\right)} \frac{K_{0} M_{\gamma_{i}}(1-\underline{v} \omega)}{\omega} \frac{1}{1-\hat{e}_{\ominus_{\nu} \omega}(\theta, 0)} \rightarrow 0 .
$$

Hence

$$
\lim _{r \rightarrow \infty} \frac{1}{m\left(r, \rho, t_{0}\right)} \int_{t_{0}-r}^{t_{0}+r}\left\|\Upsilon_{2}(t)\right\| \nabla t=0
$$

Thus, $\sum_{t_{i}<\cdot} T\left(\cdot, t_{i}\right) I_{i}\left(\vartheta\left(t_{i}\right)\right) \in P A A_{0}(\mathbb{T}, \rho)$, then $F(\cdot) \in W P A A(\mathbb{T}, \rho)$. This completes the proof.

Lemma 4.5 If $x \in P C_{\mathrm{ld}}\left(\mathbb{T}, \mathbb{R}^{+}\right)$satisfies the following inequality:

$$
x(t) \leq \alpha+\int_{(a, t]_{\mathbb{T}}} p_{1}(\tau) x(\tau) \nabla \tau+\sum_{t_{k}<t} \beta_{k} x\left(t_{k}\right), \quad \forall t \in \mathbb{T},
$$

then

$$
x(t) \leq \alpha \prod_{t_{k}<t}\left(1+\beta_{k}\right) \hat{e}_{p_{1}}(t, a), \quad \forall t \in \mathbb{T} .
$$

Proof Define

$$
r(t)=\alpha+\int_{(a, t]_{\mathbb{T}}} p_{1}(\tau) x(\tau) \nabla \tau+\sum_{t_{k}<t} \beta_{k} x\left(t_{k}\right), \quad \forall t \in \mathbb{T} .
$$

Consider

$$
\left\{\begin{array}{l}
r^{\nabla}(t)=p_{1}(t) x(t) \leq p_{1}(t) r(t), \quad t \neq t_{k}, \\
r(a)=\alpha, \quad r\left(t_{k}^{+}\right)=\left(1+\beta_{k}\right) r\left(t_{k}\right) .
\end{array}\right.
$$

For $t \in\left(a, t_{1}\right]_{\mathbb{T}}$, we can calculate that

$$
\begin{aligned}
{\left[r \hat{e}_{\ominus_{\nu} p_{1}}\left(\cdot, t_{0}\right)\right]^{\nabla} } & =r^{\nabla}(t) e_{\ominus_{\nu} p_{1}}\left(\varrho(t), t_{0}\right)+r(t)\left(\ominus_{\nu} p_{1}\right)(t) \hat{e}_{\ominus_{v} p_{1}}\left(t, t_{0}\right) \\
& =r^{\nabla}(t) e_{\ominus_{\nu} p_{1}}\left(\varrho(t), t_{0}\right)+r(t) \frac{\left(\ominus p_{1}\right)(t)}{1-v(t)\left(\ominus p_{1}\right)(t)} \hat{e}_{\ominus_{\nu} p_{1}}\left(\varrho(t), t_{0}\right) \\
& =\left[r^{\nabla}(t)-\left(\ominus_{\nu}\left(\ominus_{\nu} p_{1}\right)\right)(t) r(t)\right] \hat{e}_{\ominus_{\nu} p_{1}}\left(\varrho(t), t_{0}\right) \\
& =\left[r^{\nabla}(t)-p_{1}(t) r(t)\right] \hat{e}_{\ominus_{\nu} p_{1}}\left(\varrho(t), t_{0}\right) \\
& \leq 0 .
\end{aligned}
$$


This implies that $x(t) \leq \alpha \hat{e}_{p_{1}}(t, a)$. Further we have

$$
\begin{aligned}
r(t) & \leq r\left(t_{i}^{+}\right) \hat{e}_{p_{1}}\left(t, t_{i}\right) \leq \alpha\left(1+\beta_{i}\right) \prod_{t_{k}<t_{i}}\left(1+\beta_{k}\right) \hat{e}_{p_{1}}\left(t_{i}, a\right) \hat{e}_{p_{1}}\left(t, t_{i}\right) \\
& =\alpha \prod_{t_{k}<t}\left(1+\beta_{k}\right) \hat{e}_{p_{1}}(t, a), \quad t \in\left(t_{i}, t_{i+1}\right]_{\mathbb{T}} .
\end{aligned}
$$

Thus

$$
x(t) \leq \alpha \prod_{t_{k}<t}\left(1+\beta_{k}\right) \hat{e}_{p_{1}}(t, a), \quad t \in \mathbb{T} .
$$

This completes the proof.

The following existence result is based on the contraction principle.

Theorem 4.6 Assume the following conditions hold:

$\left(\mathrm{A}_{1}\right)$ The family $\{A(t): t \in \mathbb{T}\}$ of operators in $\mathbb{X}$ generates an exponentially stable evolution system $\{T(t, s): t \geq s\}$, i.e., there exist $K_{0}>1$ and $\omega>0$ such that

$$
\|T(t, s)\|_{B(\mathbb{X})} \leq K_{0} \hat{e}_{\ominus_{\nu} \omega}(t, s), \quad t \geq s,
$$

and for any sequence $\left\{s_{n}\right\} \subset \Pi$, there exists a subsequence $\left\{s_{n}^{\prime}\right\} \subset\left\{s_{n}\right\}$ such that

$$
\lim _{n \rightarrow \infty} T\left(t+s_{n}^{\prime}, s+s_{n}^{\prime}\right)=T^{*}(t, s) \text { is well defined for each } t, s \in \mathbb{T}, t \geq s .
$$

$\left(\mathrm{A}_{2}\right) f \in W P A A(\mathbb{T} \times \Omega, \rho)$ and $f$ satisfies the Lipschitz condition with respect to the second argument, i.e.,

$$
\|f(t, x)-f(t, y)\| \leq L_{1}\|x-y\|, \quad t \in \mathbb{T}, x, y \in \Omega .
$$

$\left(\mathrm{A}_{3}\right) I_{i}$ is a weighted pseudo almost periodic sequence, and there exists a number $L_{2}>0$ such that

$$
\left\|I_{i}(x)-I_{i}(y)\right\| \leq L_{2}\|x-y\|
$$

for all $x, y \in \Omega, i \in \mathbb{Z}$.

Assume that

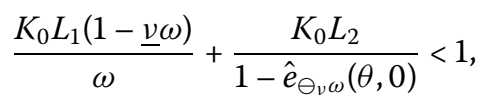

then Eq. (1) has a unique weighted piecewise pseudo almost automorphic mild solution.

Proof Consider the nonlinear operator $\Gamma$ given by

$$
\Gamma \varphi=\int_{-\infty}^{t} T(t, s) f(s, \varphi(s)) \nabla s+\sum_{t_{i}<t} T\left(t, t_{i}\right) I_{i}\left(\varphi\left(t_{i}\right)\right) .
$$

By Theorem 4.4, we see that $\Gamma$ maps $\operatorname{WPA}(\mathbb{T}, \rho)$ into $W P A A(\mathbb{T}, \rho)$. 
It suffices now to show that the operator $\Gamma$ has a fixed point in $\operatorname{WPAA}(\mathbb{T}, \rho)$. For $\varphi_{1}, \varphi_{2} \in$ $\operatorname{WPAA}(\mathbb{T}, \rho)$, one has the following:

$$
\begin{aligned}
\left\|\Gamma \varphi_{1}(t)-\Gamma \varphi_{2}(t)\right\|= & \| \int_{-\infty}^{t} T(t, s)\left[f\left(s, \varphi_{1}(s)\right)-f\left(s, \varphi_{2}(s)\right)\right] \nabla s \\
& +\sum_{t_{i}<t} T\left(t, t_{i}\right)\left[I_{i}\left(\varphi_{1}\left(t_{i}\right)\right)-I_{i}\left(\varphi_{2}\left(t_{i}\right)\right)\right] \| \\
\leq & \int_{-\infty}^{t} K_{0} \hat{e}_{\ominus_{\nu} \omega}(t, s)\left\|f\left(s, \varphi_{1}(s)\right)-f\left(s, \varphi_{2}(s)\right)\right\| \nabla s \\
& +\sum_{t_{i}<t} K_{0} \hat{e}_{\ominus_{\nu} \omega}\left(t, t_{i}\right)\left\|I_{i}\left(\varphi_{1}\left(t_{i}\right)\right)-I_{i}\left(\varphi_{2}\left(t_{i}\right)\right)\right\| \\
\leq & \int_{-\infty}^{t} K_{0} \hat{e}_{\ominus_{\nu} \omega}(t, s) L_{1}\left\|\varphi_{1}(s)-\varphi_{2}(s)\right\| \nabla s \\
& +\sum_{t_{i}<t} K_{0} \hat{e}_{\ominus_{\nu} \omega}\left(t, t_{i}\right) L_{2}\left\|\varphi_{1}\left(t_{i}\right)-\varphi_{2}\left(t_{i}\right)\right\| \\
\leq & {\left[\frac{K_{0} L_{1}(1-\underline{v} \omega)}{\omega}+\frac{K_{0} L_{2}}{1-\hat{e}_{\ominus_{v} \omega}(\theta, 0)}\right]\left\|\varphi_{1}-\varphi_{2}\right\| . }
\end{aligned}
$$

Since $\frac{K_{0} L_{1}(1-\underline{\underline{v} \omega)}}{\omega}+\frac{K_{0} L_{2}}{1-\hat{e}_{\ominus} \omega(\theta, 0)}<1, \Gamma$ is a contradiction. Hence, $\Gamma$ has a fixed point in $\operatorname{WPAA}(\mathbb{T}, \rho)$, then Eq. (1) has a unique weighted piecewise pseudo almost automorphic mild solution. This completes the proof.

Finally, we investigate the exponential stability of a weighted piecewise pseudo almost automorphic mild solution to Eq. (1). By using the Gronwall-Bellman inequality (see Lemma 4.5) and the Lipschitz condition, it can be formulated as follows.

Theorem 4.7 Suppose the conditions of Theorem 4.6 hold. Assume further that $\left(\ominus_{\nu} \omega\right) \oplus_{v}$ $p<0$, where $p=\frac{K_{0} L_{1}}{1-\bar{\nu} \omega}, \bar{v}=\sup _{t \in \mathbb{T}} v(t)$. Then Eq. (1) has a weighted piecewise pseudo almost automorphic mild solution which is exponentially stable.

Proof By Theorem 4.6, we know that Eq. (1) has a weighted piecewise pseudo almost automorphic mild solution $u(t)$, by using integral form of Eq. (1):

$$
u(t)=T\left(t, s_{0}\right) u\left(s_{0}\right)+\int_{s_{0}}^{t} T(t, s) f(s, u(s)) \nabla s+\sum_{s_{0}<t_{i}<t} T\left(t, t_{i}\right) I_{i}\left(u\left(t_{i}\right)\right),
$$

where $t>s_{0}, s_{0} \neq t_{i}, i \in \mathbb{Z}$.

Let $u(t)=u\left(t, s_{0}\right)$ and $v(t)=v\left(t, s_{0}\right)$ be two solutions of Eq. (1), then

$$
\begin{aligned}
& u(t)=T\left(t, s_{0}\right) u\left(s_{0}\right)+\int_{s_{0}}^{t} T(t, s) f(s, u(s)) \nabla s+\sum_{s_{0}<t_{i}<t} T\left(t, t_{i}\right) I_{i}\left(u\left(t_{i}\right)\right), \\
& v(t)=T\left(t, s_{0}\right) v\left(s_{0}\right)+\int_{s_{0}}^{t} T(t, s) f(s, v(s)) \nabla s+\sum_{s_{0}<t_{i}<t} T\left(t, t_{i}\right) I_{i}\left(v\left(t_{i}\right)\right) .
\end{aligned}
$$


Hence,

$$
\begin{aligned}
\|u(t)-v(t)\| \leq & \left\|T\left(t, s_{0}\right)\left[u\left(s_{0}\right)-v\left(s_{0}\right)\right]\right\| \\
& +\left\|\int_{s_{0}}^{t} T(t, s)[f(s, u(s))-f(s, v(s))] \nabla s\right\| \\
& +\left\|\sum_{s_{0}<t_{i}<t} T\left(t, t_{i}\right)\left[I_{i}\left(u\left(t_{i}\right)\right)-I_{i}\left(v\left(t_{i}\right)\right)\right]\right\| \\
\leq & \left\|T\left(t, s_{0}\right)\right\|\left\|u\left(s_{0}\right)-v\left(s_{0}\right)\right\| \\
& +\int_{s_{0}}^{t}\|T(t, s)\|\|f(s, u(s))-f(s, v(s))\| \nabla s \\
& +\sum_{s_{0}<t_{i}<t}\left\|T\left(t, t_{i}\right)\right\|\left\|\left[I_{i}\left(u\left(t_{i}\right)\right)-I_{i}\left(v\left(t_{i}\right)\right)\right]\right\| \\
\leq & K_{0} \hat{e}_{\ominus_{v} \omega}\left(t, s_{0}\right)\left\|u\left(s_{0}\right)-v\left(s_{0}\right)\right\| \\
& +\int_{s_{0}}^{t} K_{0} L_{1} \hat{e}_{\ominus v}(t, s)\|u(s)-v(s)\| \nabla s \\
& +\sum_{s_{0}<t_{i}<t} K_{0} L_{2} \hat{e}_{\ominus_{v} \omega}\left(t, t_{i}\right)\left\|u\left(t_{i}\right)-v\left(t_{i}\right)\right\| .
\end{aligned}
$$

Let $y(t)=\|u(t)-v(t)\| \hat{e}_{\omega}\left(t, t_{0}\right)$ and it follows that

$$
y(t) \leq K_{0} y\left(s_{0}\right)+K_{0} L_{1} \frac{1}{1-\bar{v} \omega} \int_{s_{0}}^{t} y(s) \nabla s+K_{0} L_{2} \sum_{s_{0}<t_{i}<t} y\left(t_{i}\right),
$$

from Lemma 4.5, one has

$$
y(t) \leq K_{0} y\left(s_{0}\right) \prod_{s_{0}<t_{i}<t} K_{0} L_{2} \hat{e}_{p}\left(t, t_{0}\right)
$$

or

$$
\|u(t)-v(t)\| \leq K_{0}\left\|u\left(s_{0}\right)-v\left(s_{0}\right)\right\| \prod_{s_{0}<t_{i}<t} K_{0} L_{2} \hat{e}_{p}\left(t, t_{0}\right) \hat{e}_{\left(\ominus_{v} \omega\right) \oplus_{v} p}\left(t, t_{0}\right),
$$

where

$$
p=\frac{K_{0} L_{1}}{1-\bar{v} \omega} .
$$

Hence, Eq. (1) has a weighted piecewise pseudo almost automorphic mild solution which is exponentially stable. This completes the proof.

Example 4.8 Let $\mathbb{T}$ be an almost periodic time scale with $v<\frac{5}{3}$ and $u: \mathbb{T} \times \mathbb{T} \rightarrow \mathbb{R}$. Consider the following problem:

$$
\left\{\begin{array}{l}
\frac{\partial}{\nabla_{1} t} u(t, x)=\frac{\partial^{2}}{\nabla_{2} x^{2}} u(\varrho(t), x)+\frac{1}{18}(\sin t+\sin \sqrt{2} t+g(t)) \cos u(t, x), \\
\quad t, x \in \mathbb{T}, t \neq t_{i}, x \in[0, \pi]_{\mathbb{T}}, \\
\Delta u\left(t_{i}, x\right)=\beta_{i} u\left(t_{i}, x\right), \quad i \in \mathbb{Z}, x \in[0, \pi]_{\mathbb{T}}, \\
u(t, 0)=u(t, \pi)=0, \quad t \in \mathbb{T},
\end{array}\right.
$$


where $g \in U P C(\mathbb{T}, \mathbb{R})$ satisfies $|g(t)| \leq 1(t \in \mathbb{T})$ and $\rho(t)=|\sin t|+1, \beta_{i}=\frac{1}{18}(\sin i+\sin \sqrt{2} i+$ $g(i))$ and $t_{i}=i+\frac{1}{8}|\sin i-\sin \sqrt{2} i|, i \in \mathbb{Z}$.

Define $\mathbb{X}=L^{2}[0, \pi]_{\mathbb{T}}$, let $A u=\frac{\partial^{2}}{\nabla_{2} x^{2}} u(t, x), u \in D(A)=H_{0}^{1}[0, \pi]_{\mathbb{T}} \cap H^{2}[0, \pi]_{\mathbb{T}}$. Clearly, it follows from the same discussion as in Section 3.1 in [50] that one can easily see that the evolution system $\{T(t, s): t \geq s\}$ satisfies $\|T(t, s)\| \leq \hat{e}_{\ominus_{v} \frac{1}{2}}(t, s)(t \geq s)$ with $K_{0}=1, \omega=1 / 2$. Furthermore, by Definition 3.3, it is easy to check that $\left\{t_{i}^{j}\right\}, i, j \in \mathbb{Z}$, is an equipotentially almost automorphic sequence and

$$
\begin{aligned}
t_{i}^{1} & =t_{i+1}-t_{i}=1+\frac{1}{8}|\sin (i+1)-\sin \sqrt{2}(i+1)|-\frac{1}{8}|\sin i-\sin \sqrt{2} i| \\
& \geq 1-\frac{1}{8}|\sin (i+1)-\sin i-[\sin \sqrt{2}(i+1)-\sin \sqrt{2} i]| \\
& \geq 1-\frac{1}{4}\left|\sin \frac{1}{2} \cos \frac{2 i+1}{2}\right|-\frac{1}{2}\left|\sin \frac{\sqrt{2}}{2} \cos \frac{\sqrt{2}(2 i+1)}{2}\right| \\
& \geq 1-\frac{1}{4} \sin \frac{1}{2}-\frac{1}{2} \sin \frac{\sqrt{2}}{2}>\frac{2}{5} .
\end{aligned}
$$

Hence, $\theta=\inf _{i \in \mathbb{Z}}\left(t_{i+1}-t_{i}\right)>\frac{2}{5}>0$. Let $f(t, u)=\frac{1}{18}(\sin t+\sin \sqrt{2} t+g(t)) \cos u, I_{i}(u)=\beta_{i} u$. Clearly, both $f$ and $I_{i}$ satisfy the assumptions given in Theorem 4.6 and Theorem 4.7 with $L_{1}=L_{2}=\frac{1}{6}$. Moreover,

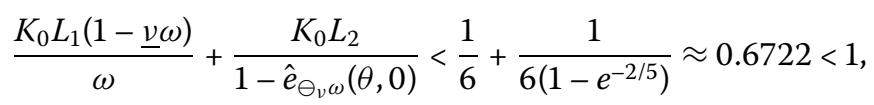

and since $v<\frac{5}{3}$, one has $1-v \omega>\frac{1}{6}$, so

$$
\left(\ominus_{\nu} \omega\right) \oplus_{\nu} p=-\frac{\omega}{1-\nu \omega}+\frac{K_{0} L_{1}}{1-\bar{v} \omega}+\frac{K_{0} L_{1} \omega \nu}{(1-\nu \omega)(1-\underline{v} \omega)} \approx-0.8333<0 .
$$

Therefore, Eq. (12) has a weighted piecewise pseudo almost automorphic mild solution which is exponentially stable.

\section{Conclusion and further discussion}

In this paper, the concept of weighted piecewise pseudo almost automorphic functions on time scales is introduced and discussed. It is well known that the $\Delta$-dynamic equations are more popular in the references, however, $\nabla$-dynamic equations are also interesting in both theory and practice. Therefore, we choose to investigate the weighted piecewise pseudo almost automorphic mild solutions to Eq. (1). All obtained results are essentially new.

Moreover, Definition 4.3 gives the expression of mild solutions to Eq. (1). It is worth emphasizing that the methods used in this paper can also be applied to study the weighted piecewise pseudo almost automorphic mild solutions to other abstract impulsive $\nabla$-dynamic equations and $\Delta$-dynamic equations. Now, similar to the discussion below Definition 4.3, we can list the mild solutions to three representative classes of abstract impulsive dynamic equations on time scales. 
Type (i). Consider a $\nabla$-dynamic equation as follows:

$$
\left\{\begin{array}{l}
x^{\nabla}(t)=A(t) x(t)+f(t, x(t)), \quad t \in \mathbb{T}, t \neq t_{i}, i \in \mathbb{Z}, \\
\Delta x\left(t_{i}\right)=x\left(t_{i}^{+}\right)-x\left(t_{i}^{-}\right)=I_{i}\left(x\left(t_{i}\right)\right), \quad t=t_{i},
\end{array}\right.
$$

where $A \in P C_{\mathrm{ld}}(\mathbb{T}, \mathbb{X})$ is a linear operator in the Banach space $\mathbb{X}$ and $f \in P C_{\mathrm{ld}}(\mathbb{T} \times \mathbb{X}, \mathbb{X})$. Then, for any $t \in \mathbb{T}, t>c, c \neq t, i \in \mathbb{Z}$, Eq. (13) has the following mild solution:

$$
x(t)=T(t, c) x(c)+\int_{c}^{t} T(t, \varrho(s)) f(s, x(s)) \nabla s+\sum_{c<t_{i}<t} T\left(t, t_{i}\right) I_{i}\left(x\left(t_{i}\right)\right) .
$$

Type (ii). Consider a $\Delta$-dynamic equation as follows:

$$
\left\{\begin{array}{l}
x^{\Delta}(t)=A(t) x^{\sigma}+f(t, x(t)), \quad t \in \mathbb{T}, t \neq t_{i}, i \in \mathbb{Z}, \\
\Delta x\left(t_{i}\right)=x\left(t_{i}^{+}\right)-x\left(t_{i}^{-}\right)=I_{i}\left(x\left(t_{i}\right)\right), \quad t=t_{i},
\end{array}\right.
$$

where $A \in P C_{\mathrm{rd}}(\mathbb{T}, \mathbb{X})$ is a linear operator in the Banach space $\mathbb{X}$ and $f \in P C_{\mathrm{rd}}(\mathbb{T} \times \mathbb{X}, \mathbb{X})$, $x^{\sigma}=x(\sigma(t))$. Then, for any $t \in \mathbb{T}, t>c, c \neq t_{i}, i \in \mathbb{Z}$, Eq. (14) has the following mild solution:

$$
x(t)=T(t, c) x(c)+\int_{c}^{t} T(t, s) f(s, x(s)) \Delta s+\sum_{c<t_{i}<t} T\left(t, t_{i}\right) I_{i}\left(x\left(t_{i}\right)\right) .
$$

Type (iii). Consider a $\Delta$-dynamic equation as follows:

$$
\left\{\begin{array}{l}
x^{\Delta}(t)=A(t) x(t)+f(t, x(t)), \quad t \in \mathbb{T}, t \neq t_{i}, i \in \mathbb{Z}, \\
\Delta x\left(t_{i}\right)=x\left(t_{i}^{+}\right)-x\left(t_{i}^{-}\right)=I_{i}\left(x\left(t_{i}\right)\right), \quad t=t_{i},
\end{array}\right.
$$

where $A \in P C_{\mathrm{rd}}(\mathbb{T}, \mathbb{X})$ is a linear operator in the Banach space $\mathbb{X}$ and $f \in P C_{\mathrm{rd}}(\mathbb{T} \times \mathbb{X}, \mathbb{X})$. Then, for any $t \in \mathbb{T}, t>c, c \neq t_{i}, i \in \mathbb{Z}$, Eq. (15) has the following mild solution:

$$
x(t)=T(t, c) x(c)+\int_{c}^{t} T(t, \sigma(s)) f(s, x(s)) \Delta s+\sum_{c<t_{i}<t} T\left(t, t_{i}\right) I_{i}\left(x\left(t_{i}\right)\right) .
$$

By the same discussion method in this paper, one can show that the above three mild solutions are weighted piecewise pseudo almost automorphic solutions to their corresponding abstract impulsive dynamic equations on time scales. For example, in [49], the concept of rd-piecewise continuous functions is introduced, by the same discussion method in this paper, the weighted piecewise pseudo almost automorphic mild solutions to Eq. (14) and Eq. (15) can be studied. Furthermore, all the results can be applied to study almost automorphic solutions to impulsive dynamic models such as impulsive neural networks or biological models, economical models with impulsive effects on time scales.

The authors declare that they have no competing interests. 


\section{Author details}

1 Department of Mathematics, Yunnan University, Kunming, Yunnan 650091, People's Republic of China. ${ }^{2}$ Department of Mathematics, Texas A\&M University-Kingsville, 700 University Blvd., Kingsville, TX 78363-8202, USA. ${ }^{3}$ Department of Mathematics, Faculty of Science, King Abdulaziz University, P.O. Box 80203, Jeddah, 21589, Saudi Arabia.

\section{Acknowledgements}

The authors would like to express their sincere thanks to the referees for suggesting some corrections that help making the content of the paper more accurate. This work is supported by the foundation of Yunnan University in China (No. 2013CG020).

\section{Received: 14 February 2014 Accepted: 12 May 2014 Published: 22 May 2014}

\section{References}

1. Bochner, S: Uniform convergence of monotone sequences of functions. Proc. Natl. Acad. Sci. USA 47, $582-585$ (1961)

2. Bochner, S: A new approach in almost-periodicity. Proc. Natl. Acad. Sci. USA 48, 2039-2043 (1962)

3. Bochner, S: Continuous mappings of almost automorphic and almost periodic functions. Proc. Natl. Acad. Sci. USA 52, 907-910 (1964)

4. N'Guérékata, GM: Topics in Almost Automorphy. Springer, New York (2005)

5. Diagana, T: Almost Automorphic Type and Almost Periodic Type Functions in Abstract Spaces. Springer, Cham (2013)

6. N'Guérékata, GM: Almost Automorphic and Almost Periodic Functions in Abstract Spaces. Plenum, New York (2001)

7. Caraballo, T, Cheban, D: Almost periodicity and automorphic solutions of linear differential/difference equations without Favard's separation condition I. J. Differ. Equ. 246, 108-128 (2009)

8. Caraballo, T, Cheban, D: Almost periodicity and automorphic solutions of linear differential/difference equations without Favard's separation condition II. J. Differ. Equ. 246, 1164-1186 (2009)

9. Caraballo, T, Cheban, D: Levitan/Bohr almost periodic and almost automorphic solutions of second order monotone differential equations. J. Differ. Equ. 251, 708-727 (2011)

10. Cieutat, P, Ezzinbi, K: Almost automorphic solutions for some evolution equations through the minimizing for some subvariant functional, applications to heat and wave equations with nonlinearities. J. Funct. Anal. 260, 2598-2634 (2011)

11. Goldstein, JA, N'Guérékata, GM: Almost automorphic solutions of semilinear evolution equations. Proc. Am. Math Soc. 133, 2401-2408 (2005)

12. N'Guérékata, GM: Existence and uniqueness of almost automorphic mild solutions to some semilinear abstract differential equations. Semigroup Forum 69, 80-86 (2004)

13. Ezzinbi, K, Fatajou, S, N'Guérékata, GM: Pseudo almost automorphic solutions to some neutral partial functional differential equations in Banach spaces. Nonlinear Anal., Theory Methods Appl. 70, 1641-1647 (2009)

14. Diagana, T: Almost automorphic solutions to some damped second-order differential equations. Commun. Nonlinear Sci. Numer. Simul. 17, 4074-4084 (2012)

15. Ding, H, Liang, J, Xiao, T: Almost automorphic solutions to nonautonomous semilinear evolution equations in Banach spaces. Nonlinear Anal., Theory Methods Appl. 73, 1426-1438 (2010)

16. Zhao, Z, Chang, Y, Nieto, JJ: Almost automorphic and pseudo-almost automorphic mild solutions to an abstract differential equation in Banach spaces. Nonlinear Anal., Theory Methods Appl. 72, 1886-1894 (2010)

17. Sakthivel, R, Revathi, P, Marshal Anthoni, S: Existence of pseudo almost automorphic mild solutions to stochastic fractional differential equations. Nonlinear Anal., Theory Methods Appl. 75, 3339-3347 (2012)

18. Lizama, C, Mesquita, JG: Almost automorphic solutions of non-autonomous difference equations. J. Math. Anal. Appl. 407, 339-349 (2013)

19. Diagana, T: Existence of globally attracting almost automorphic solutions to some nonautonomous higher-order difference equations. Appl. Math. Comput. 219, 6510-6519 (2013)

20. Abbas, $S, X i a, Y$ : Existence and attractivity of $k$-almost automorphic sequence solution of a model of cellular neural networks with delay. Acta Math. Sci. 33, 290-302 (2013)

21. Xiao, T, Liang, J, Zhang, J: Pseudo almost automorphic solutions to semilinear differential equations in Banach spaces. Semigroup Forum 76, 518-524 (2008)

22. Blot, J, Mophou, JM, N'Guérékata, GM, Pennequin, D: Weighted pseudo almost automorphic functions and applications to abstract differential equations. Nonlinear Anal., Theory Methods Appl. 71, 903-909 (2009)

23. Diagana, T: Existence of weighted pseudo-almost periodic solutions to some classes of nonautonomous partial evolution equations. Nonlinear Anal., Theory Methods Appl. 74, 600-615 (2011)

24. Hacene, NB, Ezzinbi, K: Weighted pseudo almost periodic solutions for some partial functional differential equations. Nonlinear Anal., Theory Methods Appl. 71, 3612-3621 (2009)

25. Diagana, T: Weighted pseudo-almost periodic solutions to some differential equations. Nonlinear Anal., Theory Methods Appl. 68, 2250-2260 (2008)

26. Ding, H, Liang, J, Xiao, T: Weighted pseudo almost periodic functions and applications to evolution equations with delay. Appl. Math. Comput. 219, 8949-8958 (2013)

27. Abbas, S: Weighted pseudo almost automorphic sequences and their applications. Electron. J. Differ. Equ. 2010,12 (2010)

28. Ding, H, Liang, J, Xiao, T: Weighted pseudo almost automorphic functions and WPAA solutions to semilinear evolution equations. J. Math. Anal. Appl. 409, 409-427 (2014)

29. Mishra, I, Bahuguna, D: Weighted pseudo almost automorphic solution of an integro-differential equation, with weighted Stepanov-like pseudo almost automorphic forcing term. Appl. Math. Comput. 219, 5345-5355 (2013)

30. Chang, Y, Zhang, R, N'Guérékata, GM: Weighted pseudo almost automorphic mild solutions to semilinear fractional differential equations. Comput. Math. Appl. 64, 3160-3170 (2012)

31. Hilger, S: Ein Maßkettenkalkäul mit Anwendung auf Zentrumsmannigfaltigkeiten. PhD thesis, Universität Wäurzburg (1988)

32. Li, Y, Wang, C: Uniformly almost periodic functions and almost periodic solutions to dynamic equations on time scales. Abstr. Appl. Anal. 2011, Article ID 341520 (2011) 
33. Li, Y, Wang, C: Almost periodic functions on time scales and applications. Discrete Dyn. Nat. Soc. 2011, Article ID $727068(2011)$

34. Wang, C, Li, Y: Weighted pseudo almost automorphic functions with applications to abstract dynamic equations on time scales. Ann. Pol. Math. 108, 225-240 (2013)

35. Lizama, C, Mesquita, JG: Almost automorphic solutions of dynamic equations on time scales. J. Funct. Anal. 265 2267-2311 (2013)

36. Bainov, DD, Simeonov, PS: Theory of Impulsive Differential Equations, Periodic Solutions and Applications. Longman, Harlow (1993)

37. Samoilenko, AM, Perestyuk, NA: Impulsive Differential Equations. World Scientific, Singapore (1995)

38. Wang, C, Li, Y, Fei, Y: Three positive periodic solutions to nonlinear neutral functional differential equations with impulses and parameters on time scales. Math. Comput. Model. 52, 1451-1462 (2010)

39. Federson, M, Mesquita, JG, Slavík, A: Measure functional differential equations and functional dynamic equations on time scales. J. Differ. Equ. 252, 3816-3847 (2012)

40. Federson, M, Mesquita, JG, Slavík, A: Basic results for functional differential and dynamic equations involving impulses. Math. Nachr. 286, 181-204 (2013)

41. Stamov, GT, Alzabut, JO: Almost periodic solutions for abstract impulsive differential equations. Nonlinear Anal., Theory Methods Appl. 72, 2457-2464 (2010)

42. Bohner, M, Peterson, A: Advances in Dynamic Equations on Time Scales. Birkhäuser Boston, Boston (2003)

43. Guseinov, GS: Integration on time scales. J. Math. Anal. Appl. 285, 107-127 (2003)

44. Lakshmikantham, V, Sivasundaram, S, Kaymakcalan, B: Dynamical Systems on Measure Chains. Kluwer Academic, Boston (1996)

45. Agarwal, RP, Bohner, M, O’Regan, D, Peterson, A: Dynamic equations on time scales: a survey. J. Comput. Appl. Math. 285, 1-26 (2006)

46. Lakshmikantham, V, Devi, JV: Hybrid systems with time scales and impulses. Nonlinear Anal., Theory Methods Appl. 65, 2147-2152 (2006)

47. Bohner, M, Peterson, A: Dynamic Equations on Time Scales: An Introduction with Applications. Birkhäuser, Boston (2001)

48. Li, Y, Wang, C: Pseudo almost periodic functions and pseudo almost periodic solutions to dynamic equations on time scales. Adv. Differ. Equ. 2012, 77 (2012)

49. Wang, C: Almost periodic solutions of impulsive BAM neural networks with variable delays on time scales. Commun. Nonlinear Sci. Numer. Simul. 19, 2828-2842 (2014)

50. Jackson, B: Partial dynamic equations on time scales. J. Comput. Appl. Math. 186, 391-415 (2006)

10.1186/1687-1847-2014-153

Cite this article as: Wang and Agarwal: Weighted piecewise pseudo almost automorphic functions with applications to abstract impulsive $\nabla$-dynamic equations on time scales. Advances in Difference Equations 2014, 2014:153

\section{Submit your manuscript to a SpringerOpen ${ }^{\ominus}$ journal and benefit from:}

- Convenient online submission

- Rigorous peer review

Immediate publication on acceptance

Open access: articles freely available online

- High visibility within the field

- Retaining the copyright to your article 